\title{
Effects of the Distributions of Energy or Charge Transfer Rates on Spectral Hole Burning in Pigment-Protein Complexes at Low
}

\section{Temperatures}

\author{
Nicoleta Herascu ${ }^{1}$, Somaya Ahmouda ${ }^{1}$, Rafael Picorel ${ }^{2,3}$, Michael Seibert ${ }^{3}$, Ryszard \\ Jankowiak $^{4}$ and Valter Zazubovich ${ }^{1, *}$ \\ ${ }^{1}$ Department of Physics, Concordia University, 7141 Sherbrooke Str. West, \\ Montreal, Quebec H4B 1 R6 Canada, ${ }^{2}$ Estacion Experimental Aula Dei (CSIC), Avda. \\ Montañana, 50059 Zaragoza, Spain; ${ }^{3}$ NREL, Golden, CO, USA, ${ }^{4}$ Department of \\ Chemistry, Kansas State University, Manhattan, KS, USA.
}

\begin{abstract}
Effects of the distributions of excitation energy transfer (EET) rates (homogeneous line widths) on the non-photochemical (resonant) spectral hole burning (SHB) processes in photosynthetic chlorophyll-protein complexes (reaction center [RC] and CP43 antenna of Photosystem II from spinach) are considered. It is demonstrated that inclusion of such a distribution results in somewhat more dispersive hole burning kinetics. More importantly, however, inclusion of the EET rate distributions strongly affects the dependence of the hole width on the fractional hole depth. Different types of line width distributions have been explored, including those resulting from Förster type EET between weakly interacting pigments as well as Gaussian ones, which may be a reasonable approximation for those resulting, for instance, from so-called extended Förster models. For Gaussian line width distributions it is possible to determine the parameters of both line width and tunneling parameter distributions from SHB data without a priori knowledge of any of them. Concerning more realistic asymmetric distributions, we demonstrate, using the simple example of CP43 antenna, that one can use SHB modeling to estimate electrostatic couplings between pigments and support or exclude assignment of certain pigment(s) to a particular state.
\end{abstract}

Keywords: Photosynthesis, spectroscopy, CP43, Photosystem II reaction center.

* corresponding author; vzazubov@alcor.concordia.ca 


\section{Introduction}

Spectral hole burning (SHB) ${ }^{1-3}$ possesses the well documented ability to access low-temperature excited state lifetimes ${ }^{4-8}$. In SHB and other frequency domain experiments, the widths of the zero-phonon lines (ZPL) / zero-phonon holes (ZPH) are measured, and they are inversely proportional to the excited state lifetimes ${ }^{9}$. Conventionally, a single excited state lifetime is obtained from the width (full width at half maximum, FWHM) of a spectral hole. However, since both the ZPL width and SHB yield depend on the lifetime, SHB has a strong tendency to preferentially probe the longer-lifetime (narrower line width) wing of the lifetime distribution, if such a distribution is present. This is definitely the case for protein-chlorophyll (Chl), lightharvesting complexes. The function of the pigments in these complexes is to harvest light and to transfer excitation energy to the reaction center (RC), responsible for primary charge separation. Thus, for the majority of the pigments in the complex (except for those in the RC and the lowest-energy states of the antenna complexes), the excited state lifetime is determined by the excitation energy transfer (EET) processes. Due to the diagonal and off-diagonal energy disorder, imparted by the protein environment, the EET times for a pigment at a given position within a complex or at given wavelength are subject to a distribution. Charge separation rates in the RC may be subject to a distribution as well ${ }^{10-13}$. Although qualitatively it is a common knowledge that SHB preferentially probes the slow EET rate wing of the distribution, this effect has not been explored quantitatively. Namely, to the best of our knowledge no one has discussed what errors are introduced by ignoring the EET rate distributions while interpreting the results of the SHB experiments in various systems. This is one of the goals of the present 
manuscript. Although Reinot et al. briefly considered the effects of the (Weibull) distribution of homogeneous line widths due to irregularities of pure dephasing for Alphtalocyanine in hyperquenched glassy water and ruled these effects negligible ${ }^{14}$, the distribution parameters may be very different in the case of EET in photosynthetic complexes. Note that the distribution of EET rates originates from energetic disorder in photosynthetic complexes, not from differences from molecule to molecule in fast spectral diffusion as was the case in ${ }^{14}$. In the absence of the distribution of homogeneous line widths, the hole burning process is governed by the distributions of the tunneling parameter $\lambda$, the angle between the laser polarization and the transition dipole, as well as by $\omega$-distribution reflecting non-resonant burning ${ }^{8,14}$.

The combined influence of all distributions was never explored in detail for photosynthetic complexes. Some time ago we made ${ }^{10}$ the first attempt to incorporate the homogeneous line width distribution into the SHB model of Hayes et al. ${ }^{15}$, which does not include dispersion of the tunneling rates. As was suggested by Prokhorenko and Holzwarth, the excited state lifetime (in this case reflecting the primary charge separation time) of the P680 state of the RC of plant Photosystem II (PSII RC) is subject to a broad distribution ${ }^{11}$. Employing the line width distribution corresponding to the primary charge separation rates based on photon echo results ${ }^{11}$, we were able to fit the triplet bottleneck (transient) holes using the same site distribution function (SDF) and electron-phonon coupling parameters as for persistent holes burnt at the same wavelengths ${ }^{10}$. We also demonstrated that the electron-phonon coupling for the P680/P684 states of the PSII RC (with P684 probably corresponding to a subpopulation of more intact RCs) might be much weaker than previously believed. Therefore, the impact of the line width 
distributions on the hole spectra in photosynthetic complexes can be quite significant and cannot be neglected. Since the analysis of ${ }^{10} \operatorname{did}$ not include $\lambda$ - and $\alpha$-distributions (see section 2.3 for more details), the fitting of the persistent holes with one and the same set of parameters (including the burn dose) for all holes was impossible. Thus, persistent SHB data obtained for PSII RC from spinach will be discussed in this manuscript. In the case of light harvesting complex 2 (LH2) ${ }^{16}$, we demonstrated that taking into account the line width distribution from ${ }^{17}$ does improve the agreement between spectral hole simulations and experiment. (The focus of ${ }^{16}$ was, however, on light-induced spectral diffusion, not on the line width distributions.)

One could also ask an even more ambitious question: can one reliably determine, from the SHB data, the whole EET time distribution at a given wavelength? This distribution could then be compared to theoretical predictions based on structural data, which in turn could yield additional information on transition energies in the absence of inter-pigment interactions ("site energies"), couplings of different Chls, and improved overall understanding of light-harvesting antennae. Calculations of optical spectra and EET rates between $\mathrm{Chl}$ molecules in various pigment-protein photosynthetic complexes became possible after the structures of these complexes were determined at high enough resolution by means of X-ray crystallography ${ }^{18-21}$. A number of structure-based calculations were performed previously for example on cyanobacterial Photosystem I (PSI) ${ }^{22,23}$ and PS II ${ }^{24}$, LH II antenna complex of purple bacteria ${ }^{25,26}$, and FMO ${ }^{25}$. These simulations yielded new insight into the excitonic structure of these complexes, as well as important results in terms of possible origins of the lowest-energy antenna states, EET times, and trapping times. However, additional comparisons between the theory and 
experiment are necessary for refinement of the parameters of such calculations - namely the sets of both inter-pigment coupling energies and the site energies. The conflicting information on these parameters, especially the site energies, is currently impeding the progress in matching the features of calculated and experimental spectra in most of the photosynthetic complexes. For example, in the case of the CP43 core antenna complex of PSII ${ }^{19}$, several assignments of quasi-degenerate A and B bands ${ }^{28}$ to different $\mathrm{Chl}$ molecules are possible ${ }^{29}$. In fact, by now 8 out of 13 Chls have been suggested to be the lowest energy ones, contributing to either localized or delocalized states. Any such assignment requires certain assumptions about the site energies of the Chls. The same is true for PSII RC and also for PSI, for which at least two sets of site energies are available in the literature, and different assignments of the lowest-energy antenna states are reported $^{22,23,30-33}$. It is also not clear which EET theories yield the best agreement with experiment in cases when the energy donor or acceptor or both are states delocalized over a large number of strongly-coupled molecules ${ }^{17,34,35}$. One of the ways to distinguish the “correct" chlorophyll-to-band assignments and/or EET theories would be through comparing theoretical predictions and observations concerning the excited state lifetimes (and their distributions) at various wavelengths.

One could argue that single photosynthetic complex spectroscopy (SPCS), when performed on a sufficiently large number of complexes, is capable of delivering the desired information on the EET rate distributions. In practice, however, low-temperature SPCS experiments are relatively difficult to perform, collecting statistically meaningful amounts of data takes a long time, and one often cannot be sure that SPCS results are free of systematic distortions originating from particular sample preparation procedures. For 
example, the sum of the many LH2 single complex spectra from ${ }^{36}$ does not yield the bulk excitation spectrum, which may indicate that in SPCS samples the LH2 complex do not reside in the same local environment as LH2 complexes in the bulk samples. In addition, small fluctuations of width and position of the spectral lines systematically affect the results of SPCS experiments ${ }^{37}$, increasing the line width averages beyond those corresponding to the results of SHB and even of the time-domain measurements. A detailed comparison of manifestations of the light-induced spectral diffusion in SPCS and SHB experiments in LH2 was performed in ${ }^{16}$. It was demonstrated that the fastest spectral diffusion observed in SPCS experiments is absent in SHB, suggesting, once again, dependence on sample preparation (the amorphous matrix surrounding the protein). Obviously, the distributions of EET times should also be apparent in the timedomain experiments. However, the common practice is to fit the time-domain results with a minimal number of decay exponents, see, for example ${ }^{29,38}$ for CP43 and ${ }^{12,39-41}$ for PSII RC, as well as ${ }^{42-44}$ for other complexes. Note that even in ${ }^{13,45,46}$, although the likely lifetime distributions have been reported, for instance as lifetime density maps, the fitting of the time-dependent behavior at each point still involved just several (3-6) exponents.

Consequently, it would be of considerable interest to explore if it is possible to develop a methodology of reliably extracting EET rate distributions from the SHB data. In order to achieve this goal, one would have to develop a detailed understanding of the combined influence of EET rate distributions and other factors affecting the shape of the hole spectra and their time (irradiation dose) evolution, and to learn disentangling these effects. This manuscript is a first step down this path. Following the general outline 
described above, first, the distribution of the EET rates will be incorporated into HB models ${ }^{14,47}$ on a theoretical level. The range of tunneling parameters typical for photosynthetic proteins has recently been determined ${ }^{16,48}$. Roughly, the hole burning rates are $\sim 2$ orders of magnitude lower in photosynthetic complexes than in molecular glasses (hyperquenched glassy water ${ }^{14,49,50}$, ethanol and methanol ${ }^{50}$, and odichlorobenzene ${ }^{51}$ ), which is in line with entities involved in tunneling being larger and heavier in the case of protein. All this provides the background necessary to start fitting the results of spectral hole burning experiments (whole hole shape evolution experiments as in ${ }^{31,52}$, and hole growth kinetics (HGK) measurements as in ${ }^{47,53}$ ) in photosynthetic complexes with the purpose of determining the EET rate distributions. We will also examine some EET (or charge separation ${ }^{10}$ ) rate distributions from the available literature, and analyze their agreement or disagreement with the SHB data.

\section{Experimental Methods.}

Spectral holes and hole growth kinetics (HGK) curves were measured for the lowest-energy regions of CP43 and PSII RC (6-Chl per 2 pheophytin) from spinach. Samples were isolated and purified as described in ${ }^{54}$ and ${ }^{10}$, respectively. Measurements were performed in a model A240 helium bath cryostat (Ukrainian Academy of Sciences) at $5 \mathrm{~K}$. Samples were diluted with glycerol 1:2 and placed in an Eppendorf Uvette featuring orthogonal optical paths of $10 \mathrm{~mm}$ and $2 \mathrm{~mm}$. Use of Uvette allowed absorbance (moderate OD) and fluorescence excitation (small OD to avoid reabsorption effects) measurements with the same sample. As discussed in detail in ${ }^{16}$, the material of the Uvette does not exhibit noticeable birefringence, justifying its use in measurements 
involving polarized light. The absorption spectra, measured with a Varian Cary 5000 spectrophotometer at a resolution of $0.5 \mathrm{~nm}$, were identical to those reported in ${ }^{54}$ and ${ }^{10}$, respectively. High-resolution SHB experiments were performed with Spectra-Physics/ Sirah Matisse-DS tunable dye laser (LDS688 or DCM dye) pumped with a 6W $532 \mathrm{~nm}$ solid state laser. Spectra and hole growth kinetics curves of CP43 were detected in fluorescence excitation mode with a Hamamatsu PMT / photon counter (with a AELP730 interference long-pass filter (Omega Optical) and some neutral-density and conventional long-pass filters (LOMO)), positioned at $90^{\circ}$ with respect to excitation beam. A polarization plane rotator was used to rotate the horizontally polarized light emitted by the laser by $90^{\circ}$ in order to achieve a situation where, given the geometry of the experiment, fluorescence from preferentially excited molecules was most effectively detected. High resolution spectra of PSII RC were measured in transmission mode (see section 4.4 for reasons). The excitation intensity was stabilized with a laser beam power stabilizer (BEOC) and adjusted with neutral density filters (ThorLabs). Some holeburning experiments on CP43 were performed employing detection with a Cary 5000 (Varian) at a resolution of $0.05 \mathrm{~nm}\left(\sim 1 \mathrm{~cm}^{-1}\right)$. Between the burns at different wavelengths, the holes were erased by heating the samples up to $\sim 150 \mathrm{~K}$. Thus, undesirable mutual interference of different holes was excluded. Simulations of the spectral hole burning were performed on PCs of the Pentium Dual Core class using software employed in Refs. 16 and 48).

\section{Spectral Hole Burning Taking into Account EET Rate Distributions - Theoretical}

\section{Treatment.}


As was demonstrated by Reinot et al. ${ }^{47}$ in the absence of the homogeneous line width distributions, the evolution of the shape of a non-photochemical hole burnt into the absorption spectrum of a chromophore in an amorphous host can be satisfactorily simulated for a broad range of irradiation (burning) doses only if all three of the following effects are taken into account: (a) $\omega$-distribution, which reflects the fact that spectral hole burning is possible with both resonant and non-resonant (via phonon sideband or via tails of the Lorentzian ZPL) excitation; (b) $\alpha$-distribution, which is the result of different chromophore molecules being oriented with their transition dipole moment vectors at different angles with regard to laser light polarization; and (c) is $\lambda$ distribution (of special importance), which is the distribution of the tunneling parameter $\lambda$ of two-level systems (TLS) of the amorphous matrix. Here $\lambda=d \sqrt{2 m V} / \hbar$, where $d$ is the displacement along generalized coordinate; $m$ is the effective mass of entity rearranging during the conformational change; and $V$ is the barrier height. Tunneling in TLS is responsible for non-photochemical spectral hole burning ${ }^{8}$, which is observed both in glasses ${ }^{14,47,49,51,53}$ and in proteins ${ }^{8,16,28,30,31,48,54}$. The basics of this model are described by Eqs.1-3 (from ${ }^{10,16,47}$ ):

$$
\phi\left(\lambda, \tau_{f l}\right)=\frac{\Omega_{0} \exp (-2 \lambda)}{\Omega_{0} \exp (-2 \lambda)+\tau_{f l}^{-1}},
$$

where $\tau_{f l}$ is the measured fluorescence lifetime. Equation 1 describes the SHB yield in the case of non-photochemical hole burning (the case for glasses, polymers and proteins), which enters into the SHB master equation:

$$
\begin{aligned}
D(\Omega, t)= & 1.5 \int d \omega L(\Omega-\omega) G(\omega) \int d \lambda f(\lambda) \times \\
& \times \int d \alpha \sin \alpha \cos ^{2} \alpha e^{-P \sigma \phi\left(\lambda, \tau_{f f}\right) L\left(\omega_{B}-\omega\right) t \cos ^{2} \alpha} .
\end{aligned}
$$


The latter expression describes the absorption at $\Omega$ following burning at $\omega_{B}$ with photon flux $P$ for time $t . G(\omega)$ is the SDF, describing the probabilities of encountering different zero-phonon transition frequencies prior to burning; $\sigma$ and $\phi$ are the integral absorption cross-section and hole burning quantum yield, respectively. $f(\lambda)$ is the Gaussian distribution of tunneling parameter, $\lambda$, affecting the hole burning yield, $\phi$, in Eq.1. It peaks at $\lambda_{0}$ and has a standard deviation of $\sigma_{\lambda} . \Omega_{0}=7.610^{12} \mathrm{~s}^{-147}$ is the constant prefactor in the Fermi Golden rule expression for the TLS relaxation rate for nonphotochemical hole burning. $L\left(\omega_{B}-\omega\right)$ is the single site absorption profile at the burn frequency, $\omega_{B}{ }^{10,16}$ :

$$
L\left(\omega_{B}-\omega\right)=\exp -\left[\sum_{k} S_{k}\left(2 \bar{n}_{k}+1\right)\right] \prod_{k=1,2} \sum_{R=0}^{\infty}\left(\frac{S_{k}^{R}}{R !}\right) l_{R, k}\left(\omega_{B}-\omega-R \omega_{k}\right),
$$

where $l_{R}$ are line shape functions with $R=0,1,2 \ldots$ corresponding to the zero, one-, two-, ... phonon transitions. For instance, $l_{0}$ is the Lorentzian zero-phonon line. The width of the homogeneous ZPL contributing to $L(\omega)$ is determined not by $\tau_{l}$, but by the total dephasing time $\tau_{2} .1 / \tau_{2}=1 /\left(2 \tau_{1}\right)+1 / \tau_{2}{ }^{*}$, where $\tau_{2} *$ is the pure dephasing time. The homogeneous line width is $\Gamma_{h o m}=1 / \pi c \tau_{2}$, where $\Gamma_{h o m}$ is in $\mathrm{cm}^{-1}$ and $c$ is speed of light in $\mathrm{cm} / \mathrm{s} . S_{k}, \omega_{k}$, and $\overline{\mathrm{n}}_{\mathrm{k}}$ are the Huang-Rhys factor, frequency and thermal occupation number of the k-th coupled phonon/vibration. Thermal occupation number $\left(\overline{\mathrm{n}}_{\mathrm{k}}\right)$ is temperature-dependent. The low-frequency phonon profile is assumed to be Gaussian on the ZPL side and Lorentzian on the other side. Alpha, $\alpha$, is the angle between the laser polarization and the transition dipole moment vector. 


\section{Page 11 of 51}

While taking the lifetime (energy transfer time) distributions into consideration, one has to keep in mind that in the case of NPHB, the EET time $\tau_{E E T}$ enters Eq. 1 above twice - once via the ZPL width in the homogeneous single-site spectrum $L\left(\omega_{B}-\omega, \tau_{E E T}\right)$, and also via the spectral hole burning yield, which has to be modified in the following way:

$$
\phi\left(\lambda, \tau_{E E T}\right)=\frac{\Omega_{0} \exp (-2 \lambda)}{\Omega_{0} \exp (-2 \lambda)+\tau_{f l}^{-1}+\tau_{E E T}^{-1}} .
$$

Note that in the case of the triplet bottleneck holes considered in ${ }^{10}$ for PSII RC, Equation 4 does not apply. Equation 4 reflects the fact that EET and NPHB are competing processes. EET removes the excitation from a given pigment and therefore reduces the probability of NPHB. On the other hand in the reaction center of PSII, triplet formation follows primary charge separation. Thus the triplet bottleneck hole burning yield is:

$$
\phi\left(\tau_{C S}\right)=\frac{\tau_{C S}^{-1}}{\tau_{f l}^{-1}+\tau_{C S}^{-1}},
$$

Here the yield does not depend on the tunneling parameter, $\lambda$; the latter is applicable only to persistent SHB. One could argue, though, that triplet bottleneck hole burning as reported in ${ }^{10}$ (i.e., with the persistent hole saturated before transient hole measurement) preferentially probes molecules / states not only with the largest line widths but also with the largest values of $\lambda$, as the latter are the last to burn persistently. The angular distribution still applies. Tunneling between different host conformations during NPHB does not to remove the excitation from a given pigment molecule and does not affect the charge separation yield.

Taking Eq. 4 into account, Eq.2 becomes: 


$$
\begin{gathered}
D(\Omega, t)=1.5 \int d \omega L\left(\Omega-\omega, \tau_{E E T}\right) G(\omega) \int d \lambda f(\lambda) \int d \Gamma T(\Gamma) \times \\
\times \int d \alpha \sin \alpha \cos ^{2} \alpha e^{-P \sigma \phi(\lambda, \Gamma) L\left(\omega_{B}-\omega, \tau_{E E T}\right) t \cos ^{2} \alpha}
\end{gathered}
$$

where $T(\Gamma)$ is the distribution of homogenous line widths and all other quantities are the same as above. (For the sake of simplicity, our software operates with the distributions of the homogeneous line widths $\Gamma=\left(2 \pi c \tau_{E E T}\right)^{-1}+$ [constant pure dephasing-limited width] (which is, for example, $0.03 \mathrm{~cm}^{-1}$ for $\mathrm{CP} 43$ at $5 \mathrm{~K}$ ), rather than the distributions of the lifetimes. The angular integral in Eq. 1 was treated as described in ${ }^{51}$. Namely, we took into account that

$$
F(x)=1.5 \int d \alpha \sin \alpha \cos ^{2} \alpha \cdot e^{-x \cos ^{2} \alpha}=\frac{1.5}{x}\left[\frac{\sqrt{\pi}}{2} \frac{\operatorname{erf}(\sqrt{x})}{\sqrt{x}}-e^{-x}\right],
$$

which was fitted with a polynomial for $\mathrm{x}<10$ and with $(1 / x) \sqrt{9 \pi / 16 x}$ for $\mathrm{x}>10$. Integrals over $\lambda$ and the homogeneous line width were calculated as sums with $\sim 30$ components each; the fast Fourier transform algorithm was utilized for integration over $\omega$. We assumed that there is no correlation between the SHB rate $\Omega_{0} \exp (-2 \lambda)$ (governed by the protein energy landscape parameters) and the energy transfer time (governed by the interpigment interactions and spectral overlaps). This means that the probability of a certain combination of $\Gamma$ and $\lambda$ is proportional to $f(\lambda) \cdot T(\Gamma)$. In complicated enough excitonically coupled systems with multiple EET pathways, depending on particular realizations of disorder, the latter assumption may not always be valid. Namely, certain states may be dominated, even at the same fixed wavelength, by different Chls in different complexes, which may exhibit distinct distributions of HB yields and transfer excitation energy along distinct paths characterized by distinct distributions of EET rates. 
To handle the cases of quasi-degenerate states, such as in $\mathrm{CP} 43^{28}$, our hole burning modeling software is capable of simulating simultaneous burning into two overlapping bands. Introducing more bands may be not justified, as it results in a significant increase in calculation time and in the number of free parameters.

As discussed in ${ }^{16}$, oscillator strength distributions, resulting from disorder for states having appreciable excitonic character, do not affect hole burning significantly. Therefore, for the sake of simplicity, only the average (center of mass in case of asymmetric distributions) values of integrated cross-section will be employed in the modeling below. The integrated absorption cross-section of an isolated Chl $a$ oriented with its transition dipole moment along the electric field of the incoming radiation was estimated as $4.5 \cdot 10^{-13} \mathrm{~cm}^{2} \mathrm{~cm}^{-1}$. This estimate is based on the Chl $a$ molar extinction coefficients in methanol and diethyl ether ${ }^{55}$. The $\mathrm{cm}^{2} \mathrm{~cm}^{-1}$ units are used because in our software the single site absorption spectrum is normalized on $\mathrm{cm}^{-1}$ scale (i.e., the units of $L(\omega)$ are $\mathrm{cm}) . \mathrm{In}^{2} \mathrm{~s}^{-1}$ units our integrated cross-section estimates are equivalent to $1.6 \cdot 10^{-2}$, which could be compared to $1.7510^{-2}$ reported for Al-Phthalocyanine (APT) in hyperquenched glassy water ${ }^{49}$ or to $8 \cdot 10^{-3}$ in $o$-dichlorobenzene ${ }^{51}$. Finally, the anti-holes associated with NPHB were as a rule not considered in this manuscript. In the case of the examples presented in this manuscript, this is justified by the ZPL being orders of magnitude narrower than the anti-holes.

\section{Results and Discussion}

Before engaging in a discussion on the effects of line width distributions on (persistent) spectral hole evolution, it is instructive to briefly review the key properties of 
hole growth kinetics (HGK) curves and hole width dependences on hole depth, or irradiation dose, in the case when line width distributions are not present and the hole evolution is affected mainly by a tunneling parameter (i.e., the $\lambda$-distribution). Here we ignore possible effects of delocalization of the state being burned on the hole growth process. In the following the Huang-Rhys factor for the phonon sideband (PSB) was set to $\mathrm{S}=0.6$, the PSB peak was set at $22 \mathrm{~cm}^{-1}$, and the latter had Gaussian (Lorentzian) component widths of $20 \mathrm{~cm}^{-1}\left(70 \mathrm{~cm}^{-1}\right)$, respectively. The homogeneous ZPL width was set at $3 \mathrm{~cm}^{-1}$ (lifetime of $1.7 \mathrm{ps}$ ). Figure 1A contains dispersive HGK curves for burn wavelength $\lambda_{B}$ at the peak of the SDF (with the width of $120 \mathrm{~cm}^{-1}$; note that the parameters in this example resemble those of the B800 band of the LH2 ${ }^{16}$ ). The HGK curves are calculated using Eqs. $1-3$ for $\lambda_{0}=10$ and $\sigma_{\lambda}=0.0$ (no dispersion), 0.3, 0.6, 1.0 and 1.5 , corresponding to black, blue, red, green and purple curves, respectively. Figure 1B shows the hole FWHM as a function of the fractional hole depth (curves, from bottom to top, were generated with same tunneling distribution parameters as in frame A. The increased broadening of the holes at larger $\sigma_{\lambda}$ values is due to saturation broadening of the fraction of the hole corresponding to the smallest values of $\lambda$. The insert shows an example of an individual hole spectrum. The arrows indicate that the hole width was defined and measured at half the total hole depth, including the ZPL and phonon sideband contributions. No fitting procedure was employed. Due to this particular definition of the hole width, at zero irradiation dose the hole width in frame B extrapolates to a slightly higher value than twice the homogeneous ZPL width. Changing the mean of the tunneling parameter distribution within a realistic range of $\lambda_{0}$ typically observed in photosynthetic complexes ( 10.0 to 11.5 with $\Omega_{0}=7.6 \cdot 10^{12} \mathrm{~s}^{-1} 16,48$ ) shifts the 
curves reported in frame A into different irradiation dose ranges without a change in their shape (on a logarithmic dose scale); the curves in frame B remain practically unaffected by variations of $\lambda_{0}$ within the above range.

\subsection{Gaussian line width distribution.}

One could argue that the choice of a Gaussian shape for the line width distributions is somewhat artificial and has no direct theoretical basis. On the other hand, consideration of Gaussian distributions provides valuable insight into the behavior of spectral holes in the case of broad and monotonously changing line width distributions, such as those for the B800 band of LH2 from the work of Silbey et al. ${ }^{17}$, Scholes and Fleming ${ }^{34}$, and Sumi et al. ${ }^{35,56,57}$. Very recently Pishchalnikov et al. ${ }^{58}$ proposed a close to Gaussian distribution of the primary charge separation rates in the RC of Photosystem II. Close-to-Gaussian distributions may also result from Redfield models, if the EET rate is a slowly changing function (within the range determined by the Gaussian acceptor SDF) of the donor-acceptor energy gap ${ }^{59}$ (see the next section for more details). Figure 2A shows HGK curves obtained with fixed parameters of the tunneling distribution ( $\lambda_{0}=10.0$ and $\sigma_{\lambda}=1.0$ ), with Gaussian line width distributions peaking at $3 \mathrm{~cm}^{-1}$, and with line width distribution widths (FWHM) of 0.0 (no distribution), $0.5,1.0,2.0$ and $3.0 \mathrm{~cm}^{-1}$, corresponding to the black, blue, red, green and purple curves, respectively. The insert of Figure 2A depicts the beginning of the HGK curves on a linear scale. Comparing this figure to Figure 1A, one can easily notice that the introduction of the line width distribution has minor effect on the HGK compared to that of the tunneling parameter distribution. Figure 2B contains the dependences of the hole FWHM on fractional hole 
depth for same line width distribution parameters as in frame A. Color coding is the same as well. The black curve in Figure 2B is the same as the green curve in Figure 1B. The dashed line is at twice the mean homogeneous line width. Note that the line width distribution affects the hole width dependence in a qualitatively different manner with respect to the tunneling parameter distribution. As expected, for broad enough line width distributions, the hole widths extrapolate, at zero irradiation dose, to values significantly smaller than that determined by the mean homogeneous line width. In the situation corresponding to the magenta curve (distribution peak $=$ distribution width $=3 \mathrm{~cm}^{-1}$; close to those proposed for LH2 by Silbey, Sumi, Fleming and coworkers ${ }^{17,34,35}$ ) considering, for example, $5-10 \%$ deep holes and ignoring the line width distribution results in an $\sim 1.5$ overestimation of the excited state lifetime. This is the most likely explanation for the discrepancy in the B800 excited state lifetimes obtained in SHB and time-domain experiments. Interestingly, changing $\lambda_{0}$ does not affect the shape of the width dependence on depth significantly. Figure 2C contains HGK curves for Gaussian line width distributions peaking at $0.3 \mathrm{~cm}^{-1}$ and with distribution widths (FWHM) of 0.0 (no distribution) $0.05,0.1,0.2$ and $0.3 \mathrm{~cm}^{-1}$, corresponding to black, blue, red, green and purple curves, respectively. Figure 2D contains the hole width dependences on fractional hole depth for same parameters as in Figure 2C. As expected, changing the mean of the homogenous line width distribution by a factor of 10 results in 100 -fold increase in the burning rate, at least in the beginning of the hole burning process. A 10 -fold increase comes from the increase of the HB yield, while the other factor of 10 comes from the reduction of the width of the ZPL in $L(\omega)$. At later stages of burning, we observe a burning rate of $\sim 50$ times larger than that shown in Figure 2A. This is why the curves in 


\section{Page 17 of 51}

frame $\mathrm{C}$ have a slightly smaller slope compared to those in frame A. As expected, the slope of the hole width dependence on fractional hole depth (see Frame D) is reduced approximately 10-fold compared to data shown in frame B (note the different vertical scale in frames B and D).

These observations allow for developing a procedure to determine the parameters of both line width and $\lambda$ - distributions without pre-existing knowledge of any of them (it is only assumed that both are Gaussian). Suppose one can measure a HGK curve and a series of holes from which one can determine the hole width dependence on depth, as well as a realistic value of the Huang-Rhys factor, $S$. For the sake of simplicity, we assume a realistic signal to noise ratio of $\sim 100$. The experimental data could first be fitted under the assumption of the absence of a line width distribution. This will provide (i) a reasonably close estimate of $\sigma_{\lambda}$, (ii) the lower limit of the mean of the line width distribution, and (iii) the upper limit for the tunneling distribution mean, $\lambda_{0}$. If in fact the line width distribution is present, as discussed above, the theoretical hole width dependence on depth calculated with these parameters will deviate significantly from the experimental one. Suppose that the red solid curves in Figure 3 (calculated with $\lambda_{0}=10$ and $\sigma_{\lambda}=1$, with the mean and the width of the line width distribution both being $3 \mathrm{~cm}^{-1}$ ) represents the experimental data. Figure 3A contains indistinguishable (in the presence of experimental noise) HGK curves obtained for different combinations of $\lambda_{0}, \sigma_{\lambda}$, and the mean and width of the line width distribution, while Figure 3B exhibits easily distinguishable hole width dependences on hole depth for the same combinations of parameters as in Figure 3A. See the figure captions for parameters. Note that $\sigma_{\lambda}$ essentially does not change between different datasets, while $\lambda_{o}$ changes only a little. All 
dependences in frame B extrapolate to approximately the same width at zero depth (the first $1 \%$ of the curve can be ignored if we assume a $\mathrm{S} / \mathrm{N}$ ratio of 100 ). Thus we conclude that simultaneous fitting of both types of data can yield the parameters of both distributions.

\subsection{EET rate distributions for simple Förster-type model}

Another, qualitatively different class of homogeneous line width distributions can be obtained for a simple model describing Förster-type EET ${ }^{60}$ between relatively weakly coupled pigments with uncorrelated site distribution functions (SDF). This approach is appropriate for small antenna proteins such as $\mathrm{CP} 43^{28,61}, \mathrm{CP} 43^{\prime}{ }^{62}, \mathrm{CP} 29^{48}$, and LHCII 39 , where the majority of the pigments are weakly interacting. For instance, it has been suggested that the two lowest-energy pigments of CP43 are Chls 37 and 44 (notation by Loll ${ }^{19}$ ), with inter-pigment coupling of $5.8-7.6 \mathrm{~cm}^{-1}{ }^{63}$. Detailed discussions on the applicability of the Förster model and different variations of the Redfield model can be found in ${ }^{59,64-67}$. The expression for the EET rate based on parameters of the singlemolecule spectra can be written as:

$$
k_{E T}\left(\sim \Gamma_{\text {hom }}\right)=\frac{2 \pi V^{2}}{\hbar} \int E_{D}(E) A_{A}(E) d E
$$

where $E_{D}$ and $A_{A}$ are single-site donor emission and acceptor absorption spectra, including various vibronic contributions, normalized to unity on the energy scale, and $V$ is the inter-pigment coupling energy. Note that these are the spectra as measured rather than spectral densities ${ }^{59,64-67}$. The integral in the equation above is the spectral overlap integral. Equation 7 illustrates that, in addition to inter-pigment interaction energies, the EET rates are governed by the overlaps between the donor emission and acceptor 


\section{Page 19 of 51}

absorption spectra. A simple theory of dispersive kinetics of (low-temperature) EET resulting from the distribution of spectral overlaps has been developed by Kolaczkowski et al. ${ }^{68}$, but their approach has significant limitations. That is, some of the approximations made in ${ }^{68}$ are in fact not valid at liquid helium temperatures, where SPCS and SHB experiments are normally performed. Additionally, Kolaczkowski et al. considered, for the sake of simplicity, Gaussian distributions of either localized or delocalized phonons, which are not sufficiently good reflections of a phonon-sideband shape in a single-site spectrum. Today the parameters of electron-phonon and electron[intra-molecular vibration] coupling (phonon sideband shape, frequencies, and HuangRhys factors of intra-molecular vibrations) are sufficiently well known from SHB ${ }^{69,70}$ and Fluorescence Line Narrowing (FLN and $\triangle F L N)$ experiments ${ }^{71-73}$, and one can synthesize single site spectra and calculate relevant spectral overlaps numerically. Parameters of the single-site spectra used in subsequent simulations belong to A and B states of the CP43 complex and are summarized in Table 1. We used the frequencies and Huang-Rhys factors of the Chl $a$ intra-molecular modes as determined by Rätsep et al. ${ }^{73}$. The only exception is the $90 \mathrm{~cm}^{-1}$ mode, which does not manifest in the HB spectra of CP43. Thus, the $S$-factor for this mode has been reduced ten-fold. The shape of the phonon sideband (PSB) was determined from the SHB data on CP43 ${ }^{28,74}$. The spectral overlap dependence on donor-acceptor ZPL gap has been calculated taking into account that such dependence is essentially equal to a convolution of the absorption single-site spectra of the donor and acceptor (provided there is mirror symmetry between emission and absorption single site spectra). If the latter two spectra are normalized, the proper 
normalization of the overlap integral dependence on the D-A gap can be easily determined via

$$
\int_{0}^{\infty}\left(A_{A}(E) \otimes A_{D}(E)\right) d E=\int_{0}^{\infty} A_{A}(E) d E \times \int_{0}^{\infty} A_{D}(E) d E,
$$

where $\otimes$ denotes convolution, $A_{D}$ is the donor absorption, and other variables are as in Eq.7. In particular, if both single-site spectra are normalized to one as required by Eq. 7, the overlap integral dependence on donor-acceptor gap also will be normalized to one. Figure 4 presents the SDFs of the two bands (similar to the A [solid red curve] and B [solid blue curve] states of CP43) as well as the overlap dependence on the D-A gap (black solid curve). In addition, each SDF is divided into sub-SDF for pigments, which are (dashed curves) or are not (dotted curves) capable of downhill energy transfer. ZPLZPL contribution to overlap is located at the excitation frequency $\left(14706 \mathrm{~cm}^{-1}, 680 \mathrm{~nm}\right)$, and the peaks of the SDFs of both the A and B states are shifted by $65 \mathrm{~cm}^{-1}$ to the red $\left(14641 \mathrm{~cm}^{-1} ; 683 \mathrm{~nm}\right)$. The spectral overlap dependence based on Kolaczkowski et al. ${ }^{68}$ is presented for comparison (dash-dotted curve). It is clear that the latter curve strongly underestimates the spectral overlap and EET rates for most of the D-A gaps, except the smallest ones. As $99 \%$ of the B state SDF is located to the red with respect to the burn wavelength, $99 \%$ of the burning into the A state is affected by EET. On the other hand, only $80 \%$ of the A state SDF is located to the red with respect to burn wavelength. Consequently, $20 \%$ of burning into the B state is not affected by EET and can result in the formation of narrow, dephasing-limited holes. The overlap values for the different energy gaps were assigned probabilities according to the acceptor SDF and binned. Resulting overlap distributions, normalized to one, are presented in Figure 5. The red curve corresponds to $\mathrm{B} \rightarrow \mathrm{A}$ EET and contains a $20 \%$ contribution of the zero EET rate 


\section{Page 21 of 51}

(indicated by the dashed horizontal arrow). The blue curve corresponds to $\mathrm{A} \rightarrow \mathrm{B}$ EET. Both distributions are clearly asymmetrical. Higher values of the overlap (these would be located beyond the right edge of Fig. 5) correspond to the ZPL-ZPL contribution but are very unlikely. Note that one can use logic similar to that employed above and construct line width distributions at a fixed wavelength from the energy gap dependences of the EET rates calculated in various Redfield frameworks ${ }^{59}$. The smallest donor-acceptor gap region is where the discrepancies between the Förster and Redfield models are the largest, with the Redfield rates exceeding Förster rates by up to two orders of magnitude, if dynamic localization / polaron formation is ignored ${ }^{59}$. It is clear from our results that a possible departure from the Förster model in the small donor-acceptor ZPL gap region would not significantly affect the spectral hole burning process as the relative weight of this distribution region is small and the respective fraction of molecules burns last (see Eq. 4). If one of the SDFs were shifted to lower energies with respect to the other, the respective pigment would become the unambiguous acceptor, and the overlap distributions would be dominated by localized Chl $a$ vibrations. (This would be the case for B800-B850 EET in LH2, if B800 and B850 were single pigment molecules and this simple Förster model were applicable). The most likely overlap would be determined by two-phonon processes (the dashed arrow in Figure 4), and the maximal overlap would correspond to quasi-local chlorophyll vibrations (such as indicated by the solid arrows in Fig. 4).

The above treatment can be easily extended to the case of several competing localized acceptors. The lifetime of the excited state of the donor will be determined by the sum of the rates of energy transfer from the donor to every available acceptor. It is 
known from statistics that the probability distribution for the sum of two or more independent variables is the convolution of the distribution functions for every variable. Note that the rate distributions, not the overlap distributions, have to be convoluted. Determination of these, as well as calculation of the line widths in the one donor and one acceptor case, requires knowledge of the inter-pigment interaction energies, $V$, not just the spectral overlap distributions discussed above. These energies are obviously systemdependent, and one would have to employ some particular relatively simple photosynthetic antenna complexes to continue the discussion. In case of several independent donors with quasi-degenerate SDFs, the resulting distribution can be obtained by adding the distribution functions obtained in the previous step for every donor separately, weighted by the magnitudes of the contribution of each donor at $\omega_{B}$. Returning to one donor to one acceptor EET, two line width distributions for each burn wavelength and each interaction energy $V$ are calculated within the hole burning simulator software based on the SDF, single-site spectral parameters and the burn wavelength, and these are used for subsequent modeling of simultaneous burning into two (uncorrelated) bands.

\subsection{Application to CP43}

Figure $6 \mathrm{~A}$ depicts the absorption spectrum of $\mathrm{CP} 43$ as well as various deconvolutions suggested for the lowest-energy part of the spectrum. Jankowiak et al. ${ }^{28}$ proposed that both quasi-degenerate lowest-energy states of CP43 are localized on separate $\mathrm{Chl} a$ molecules and are not connected by fast energy transfer (A and B in Figure 6A). This view was further refined in ${ }^{54,61,63} \cdot$ In $^{75}$ it was suggested that state A is 


\section{Page 23 of 51}

delocalized over at least 3 strongly-coupled Chl molecules, while state B is localized on a single chlorophyll. In this model state A has an oscillator strength equal to that of $3 \mathrm{Chl}$ molecules and peaks at $679.8 \mathrm{~nm}$ (A3 in Figure 6A). This will be referred to as the A3B1 model in the following discussion. In contrast, Hughes et al. ${ }^{74}$ suggested that state B is the lowest-energy state of the excitonically coupled pigment system, while state A is monomeric. In the latter (“A1B3”) model, the oscillator strength of the lowest B state is approximately $1 \mathrm{Chl}$ equivalent just by coincidence, and two other excitonic states of this multimer peak at 676.6 (B" in Fig. 6A) and 681.7 nm (B' in Fig. 6A), and they have, respectively, significantly increased and significantly reduced oscillator strengths. In ${ }^{61}$ we demonstrated that, based on the shapes of the SDFs corrected for EET, one cannot distinguish between the above three possibilities, but that the requirement of slow EET between $\mathrm{A}$ and $\mathrm{B}^{28}$ is unnecessary. In ${ }^{63}$, based on extensive modeling of various nonresonant, hole-burned spectra, it was concluded that bands A and B are strongly localized on Chls 44 and 37 (Loll's notation ${ }^{19}$ ), respectively. For our EET rate / line width distribution calculations, it is important to note that the electrostatic coupling between Chls 37 and 44 is $5.8 \mathrm{~cm}^{-1} 63$ (or $7.6 \mathrm{~cm}^{-1}$ if one does not take into account the dielectric constant of the protein). This magnitude of coupling is in reasonable agreement with the EET time of $\sim 10$ ps observed for the counterparts of the A and B bands in the CP43' of PSI-CP43' supercomplex ${ }^{62}$. Below we determine if the consideration of EET distributions will provide additional arguments in favor of any of the above models.

First, in the A3B1 model, the absorption at $680 \mathrm{~nm}$ will include only A and B contributions. Thus, unless we employ an extremely broad distribution with a large fraction of very large EET rates for which there is no experimental or excitonic 
calculation basis, it is impossible to explain why the maximal achievable hole depth is only $\sim 40 \%$ (results not shown) given that the electron-phonon coupling is weak ( $\sim \sim 0.3$ ).

Figure 6B depicts the hole width dependence on the fractional hole depth. The dependence is composed of two parts: the shallow hole part was measured in fluorescence excitation mode at high resolution (black diamonds). The deeper / broader hole part of the dependence is obtained using holes registered in an absorption mode with a spectrophotometer at $0.05 \mathrm{~nm}\left(1.08 \mathrm{~cm}^{-1}\right)$ resolution (blue diamonds). The widths (and depths) of the holes extracted from the spectra measured at low resolution were corrected for spectrophotometer resolution before being plotted in Figure 6B. Apart from the deepest holes measured at high resolution and the shallowest hole measured at low resolution, the data in Fig. 6B can be fitted with a single curve. Figure 6B also depicts the theoretical width / depth dependences calculated for $V_{A B}=5 \mathrm{~cm}^{-1}$ (solid curve) and $10 \mathrm{~cm}^{-}$ ${ }^{1}$ (dashed curve). All other parameters are summarized in Table 1. The dotted curve corresponds to the fractional hole width dependence in the absence of the line width distribution. It can easily be seen that the experimental results are located between the theoretical curves for $V_{A B}=5 \mathrm{~cm}^{-1}$ and $10 \mathrm{~cm}^{-1}$. One should note that if one rules the A3B1 model unlikely, as we did above, not all of the absorption at $680 \mathrm{~nm}$ is supposed to belong to the $\mathrm{A}$ and $\mathrm{B}$ states or to be burnable. Thus, the true $\mathrm{A}+\mathrm{B}$ fractional depths corresponding to each width are larger than shown in Fig. 6B (experimental). Consequently, $V_{A B}$ is closer to $5 \mathrm{~cm}^{-1}$ than to $10 \mathrm{~cm}^{-1}$, which is in perfect agreement with our earlier assignment of Chls 44 and 37 to A and B states, respectively ${ }^{63}$. Also note that for $S=0.3$, the $39 \%$ maximum hole depth corresponds to $50 \%$ of the absorption at 680 nm belonging to burnable bands. Thus, (i) the A1B3 model of the Krausz group does not 


\section{Page 25 of 51}

appear very feasible, as in that model $>60 \%$ of absorption at $680 \mathrm{~nm}$ belongs to higher components of B (supposedly featuring very fast EET to the lowest B component) and (ii) since in the A1B1 model A and B states contribute less than $50 \%$ to the absorption, the results indicate that there is an additional state (most likely the third lowest exciton level) contributing to the absorption at $680 \mathrm{~nm}$, which features couplings to A and B pigments in the same $5-10 \mathrm{~cm}^{-1}$ range. Returning to the A1B3 model from the Krausz group ${ }^{74}$, our results still may be consistent with B being a multimer with a weak narrow upper component at $\sim 680 \mathrm{~nm}$ (i.e., with higher excitonic components not in this region, but at much higher energy ${ }^{63}$ ). This will allow for agreement with the CD data. The third B-component observed by Krausz in $\delta$-CD spectra most likely resulted either from not taking into account the anti-holes of the first two B-components, or, less likely, from an excitonic band peaking at $\sim 676 \mathrm{~nm}$, which is not part of the B-manifold and exhibits relatively slow EET to A and $\mathrm{B}$.

\subsection{Application to the Photosystem II Reaction Center}

The absorption spectrum of isolated PSII RC from spinach is presented in the Figure 7A. The main features of the spectrum are a narrow peak at $680 \mathrm{~nm}$, a broad band at $670 \mathrm{~nm}$, and a weak preparation-dependent shoulder at $\sim 684 \mathrm{~nm}$. As stated in the introduction, $\mathrm{SHB}{ }^{10}$ and photon echo ${ }^{11}$ results obtained for the lower-energy spectral region of the isolated RC of PSII may be interpreted in a consistent fashion, assuming an extremely broad distribution of charge separation times from a couple of picoseconds to several nanoseconds, although, admittedly, in recent two-dimensional (2D) electronic spectroscopy experiments on isolated PSII RC at $77 \mathrm{~K}^{13}$, a much narrower heterogeneous 
distribution of time scales associated with charge transfer was observed than the one based on photon echo results ${ }^{11}$.

In ${ }^{10}$, using less sophisticated modeling tools than are now at our disposal, we fitted the triplet bottleneck holes burnt at $684-686 \mathrm{~nm}$ and suggested that the $\sim 684 \mathrm{~nm}$ band can be attributed to the lowest-energy state of a multimer involved in charge separation in the case of less disturbed RC preparations, referred to as P684. Studies of intact oxygen-evolving PSII cores (also containing CP43 and CP47) place that state at $\sim 684-685 \mathrm{~nm}^{76}$, and suggest that isolation of the RCs results in the shift of this band to $680 \mathrm{~nm}$ (hence P680) in most, but not all, RCs. In this example we analyze if the distribution of line widths following from ${ }^{11}$ can also be used to fit the persistent holes burned at $686 \mathrm{~nm}$ in the spectrum of the PSII RC. Figure 7B shows HGK curves obtained at $686 \mathrm{~nm}$ and $5 \mathrm{~K}$ with various burn intensities, in transmission and fluorescence excitation modes. The numbers next to either the curves or the data points refer to excitation intensities in $\mathrm{mW} / \mathrm{cm}^{2}$. The data points belonging to the same "discreet" dataset were obtained by consecutive burning, measuring the hole spectrum and then burning at the same wavelength again with, in most cases, increased intensity. The fastest two HGK curves (red and magenta) were obtained in fluorescence excitation mode; other datasets were measured in transmission/absorption mode. It is clear that the hole burning rate is strongly intensity- and acquisition mode dependent. This is a manifestation of the ease with which the transient triplet bottleneck hole burning occurs in this system and an evidence for charge separation being quite probable upon excitation at $686 \mathrm{~nm}$. While the system is in the triplet state (following charge separation), it is unavailable for absorbing photons at burn wavelength - i.e., it is experiencing an analog of saturation. As a result 


\section{Page 27 of 51}

the apparent burning rate is reduced. A simple theory of triplet-related saturation, independent of the particular mechanism of the triplet state emergence, involves application of Poisson statistics. Namely, the otherwise potentially absorbable photon will really be absorbed only if it arrives at least $\tau_{\text {triplet }}$ after the previous photon, where $\tau_{\text {triplet }}$ is the triplet lifetime, which is on the order of $1 \mathrm{~ms}$ in PSII RC ${ }^{77}$. In other words, we are interested in the probability of seeing one or fewer photons in the time interval equal to the triplet lifetime for given total photon flux. This probability is described by the cumulative Poisson distribution:

$$
\rho=e^{-\bar{n}} \sum_{k=0}^{1} \frac{\bar{n}^{k}}{k !},
$$

where $\bar{n}$ is the average number of potentially absorbable photons (if triplet formation were impossible) incident on the pigment molecule per triplet lifetime. Note that the sum has only two members which have to be taken into account, for $k=0$ and 1 . Also note that $\bar{n}$ is dependent on the shape of the single site spectrum, $L(\omega)$, because resonant ZPL excitation absorption occurs much easier than for PSB excitation. Summarizing, in order to take triplet saturation-related effects into account, the exponent in Eq. 5 has to be multiplied by the probability

$$
\rho\left(\omega, \tau_{\text {triplet }}, \alpha\right)=e^{-P \sigma L(\omega) \tau_{\text {triplet }} \cos ^{2} \alpha}\left[\operatorname{P\sigma L}(\omega) \tau_{\text {triplet }} \cos ^{2} \alpha+1\right] .
$$

Here all variables are as in Eqs. 1-5. Obviously, for very short triplet lifetime or very small photon flux, $\rho \cong 1$, and no correction to Eq. 5 has to be made. Unfortunately, $\rho$ depends on the angle $\alpha$ between the laser polarization and the pigment's transition dipole, and introducing correction for saturation would negate the approximation described by Eq. 6 and require a 10- to 30-fold increase in the calculation time. Thus, we did not 
include this correction into our modeling. For this reason we did not attempt to perfect the fits to the triplet bottleneck holes from ${ }^{10}$ in this work, as the latter are likely affected not only by the line width distribution, but also by saturation effects. Note that saturation would decrease and broaden the ZPL contribution to the transient holes, causing the illusion of stronger electron-phonon coupling. Thus, our suggestion of moderate electronphonon coupling for the charge-separating state of PSII RC ${ }^{10}$ still stands.

The increased apparent hole burning rate in the fluorescence excitation mode is also in agreement with charge separation being likely upon $686 \mathrm{~nm}$ excitation: only the complexes with the slowest charge separation rates (or zero rate) contribute to fluorescence while the complexes experiencing fast charge separation do not fluoresce. In summary, to avoid saturation and to probe complexes with all possible charge separation rates, one has to apply our model to the HGK and width versus depth data obtained in the transmission mode with the lowest-possible excitation intensity. Figure 7B also contains the best fit (black solid curve) to the lowest-intensity transmission mode data set (green noisy curve + open circles). The SDF and electron-phonon coupling parameters of the 684 and $680 \mathrm{~nm}$ bands were adopted from ${ }^{10}$ and are presented in Table 2. The available values of the lifetime corresponding to the slowest depopulation of the excited state (in the absence of charge separation) range from $2 \mathrm{~ns}^{13}$ to $20 \mathrm{~ns}{ }^{12}$. We used a value of $6 \mathrm{~ns}$, in agreement with ${ }^{5,45,78}$. It is evident from the fit that for higher burn intensities, saturation effects cause discrepancies between calculations and experimental data. It can be demonstrated that the onset of the discrepancy is in agreement with the saturation described by Eqs. 9 and 10, given the photon budgets of our experiments and a triplet lifetime of $1 \mathrm{~ms}$. Figure 7C shows the experimental data and its fit for hole width vs 


\section{Page 29 of 51}

depth dependence. The (solid) curve, providing the best fit for the $686 \mathrm{~nm}$ data (open and solid circles correspond to two different complimentary datasets), was obtained with the same parameters as the theoretical HGK curve in frame B. The dashed curve was obtained using the whole distribution from ${ }^{11}$. The dotted curve was obtained without any line width distribution. It is clear that distribution from ${ }^{11}$ is not applicable to the $684 \mathrm{~nm}$ band. The more realistic distribution (resulting in the solid curve in frame C) contains only zero charge separation rate (i.e. much slower than $160 \mathrm{ps,} \mathrm{see} \mathrm{below;} \mathrm{relative} \mathrm{weight}$ $45 \%$ ) and very fast (a Gaussian peaking at $3.3 \mathrm{~cm}^{-1}[1.5 \mathrm{ps}]$, with the FWHM of $1 \mathrm{~cm}^{-1}$, relative weight 55\%) components. This Gaussian matches the slower side of the Weibull distribution used in ${ }^{10}$ to fit the triplet bottleneck holes. Concerning the zero charge separation rate component, we note that SHB is unable to distinguish between hundreds of ps charge transfer times and hundreds of ps excited state lifetimes shortened just by pure dephasing, a phenomenon present in any amorphous system ${ }^{8}$, in the absence of any charge transfer. Note that the typical $5 \mathrm{~K}$ pure dephasing-broadened homogeneous line widths are on the order of $1 \mathrm{GHz}^{28}$, which corresponds to $160 \mathrm{ps}$. Charge separation slower than that will have only minor effect on the SHB data analyzed in this work. Surprisingly, the full distribution from ${ }^{11}$ does not provide a good fit for the HB data obtained at $680 \mathrm{~nm}$ (triangles) either. The latter dataset resembles the $686 \mathrm{~nm}$ one (circles) fairly well, suggesting only a minor contribution of intermediate (tens of $\mathrm{ps}^{11,13}$ ) charge separation rates to the $680 \mathrm{~nm}$ distribution. It can also be demonstrated (results not shown) that agreement between experimental $680 \mathrm{~nm}$ data and results of simulations involving the recent charge separation rate distribution from ${ }^{58}$ is extremely poor. The presence of a fairly large fraction of RCs exhibiting very slow charge separation rates 
seems to be required to fit the hole burning data. Concerning the tens of ps contribution observed in the time-domain experiments ${ }^{11,12,13}$, we should note that SHB delivers exclusively the characteristic time of the primary charge separation process, which affects the lifetime of the excited state directly resulting from the absorption of light. Time domain methods, on the other hand, are also sensitive to secondary charge separation processes, as they obviously cause additional evolution of the spectra. Thus, taking into account that the tens of ps component has been assigned to the secondary charge separation processes ${ }^{12,13}$, its absence in our SHB data is understandable.

In summary, without going into the details of the charge separation processes in PSII RC, our results are in qualitative agreement with ${ }^{12}$, where the coexistence of two different charge separation pathways in the PSII RC at low temperature was proposed, each pathway leading to either slow (activated) or fast (activationless) charge separation. It seems plausible that the fraction of the RCs exhibiting slow charge separation could increase with the decrease of temperature. This might explain quantitative disagreement between the magnitudes of different contributions obtained at $5 \mathrm{~K}$ and at $77 \mathrm{~K}^{12,13}$. However, such a difference cannot be properly evaluated, as it is well known that the relative contributions from RC680 and RC684 very from sample to sample, and Ref. 13 did not provide $77 \mathrm{~K}$ absorption spectra for comparison with our data A detailed discussion on the charge-separation pathways is beyond the scope of this manuscript, which focused on line width distributions, but it will be attempted in future publications. We note, however, that the data presented here represents one more similarity between the hole-burning properties of the $680 \mathrm{~nm}$ and $684 \mathrm{~nm}$ states, and can be treated as further evidence in support of the P680/P684 model suggested in ${ }^{10}$. 


\section{Page 31 of 51}

\section{Conclusions:}

The effects of the distributions of EET rates on the NPHB process were explored both theoretically and experimentally in isolated CP43 antenna and isolated PSII RC from spinach. We focused on the effects of EET (or charge separation) rate distributions on persistent resonant spectral hole evolution during the burning process. Possible effects of broad Gaussian homogeneous line width distributions, as well as of the line width distributions resulting from Förster-type EET between weakly interacting pigments with uncorrelated site distribution functions have been described. It is demonstrated that while the distributions of the homogeneous line width provide only a small contribution to the dispersion of the hole-burning kinetics, their presence result in much greater dependence of the hole width on the fractional hole depth, which extrapolates (at zero dose) to values much smaller than twice the average of the line width distribution. Although at present it is impossible to reliably extract all the quantitative parameters of an arbitrary line width distribution from the SHB data alone without any pre-existing knowledge, a quantitative analysis as described in this paper can prove that such a distribution exists and allows, for example, for the extraction of approximate values of inter-pigment couplings and rejection of some band assignments.

\section{Acknowledgments:}

Authors are thankful to Dr. T. Reinot, ISU / IPRT, for fruitful discussions and for supplying the code of the program used in Refs 8, 14, 47, and to Dr. V. Prokhorenko, currently at the University of Toronto, for supplying his PSII RC charge transfer rate 
distribution from Ref. 11 in numerical form. Thomas Robert Connolly is acknowledged for his participation in writing the hole burning simulator program code. Financial support from NSERC, CFI, Concordia University and Libyan government (S.A.) is gratefully acknowledged. R.P. thanks the MICINN (Grant AGL2008-00377) and the EU FEDER Program (AGL2008-00377) in Spain, and M.S. the US Department of Energy's Photosynthetic Systems Program within the Chemical Sciences, Geoscience, and Biosciences Division of the Office of Basic Energy Sciences under NREL Contract \#DEAC36-08-GO28308 for support. R.J. acknowledges support from the NSF under grant CHE-0907958. 


\section{Page 33 of 51}

Table 1: Hole simulation parameters for CP43.

\begin{tabular}{|c|c|c|c|c|c|c|c|}
\hline $\begin{array}{l}\text { CP43 } \\
\text { band }\end{array}$ & $\begin{array}{l}\text { SDF peak; } \\
\text { width } \\
\left(\mathrm{cm}^{-1}\right)\end{array}$ & $\begin{array}{l}\text { Oscillator } \\
\text { strength } \\
\text { (Chl eq.) }\end{array}$ & $\begin{array}{l}\text { Excited } \\
\text { state } \\
\text { lifetime } \\
\text { (ns) }\end{array}$ & $\mathrm{S}_{\mathrm{PSB}}$ & $\begin{array}{c}\omega_{\mathrm{m}} ; \\
\Gamma_{\text {Gauss }} ; \\
\Gamma_{\text {Lorentz }} \\
\left(\mathrm{cm}^{-1}\right)\end{array}$ & $\lambda_{0}$ & $\sigma$ \\
\hline $\mathrm{A}$ & $\begin{array}{c}14643 \\
180\end{array}$ & 1 & 3.5 & $0.30 \pm 0.05$ & $17 ; 11 ; 70$ & $11.0 \pm 0.1$ & $1.0 \pm 0.1$ \\
\hline B & 14643,65 & 1.2 & 3.5 & $0.30 \pm 0.05$ & $24 ; 15 ; 70$ & $10.5 \pm 0.3$ & $1.0 \pm 0.1$ \\
\hline
\end{tabular}

Table 2: Hole simulation parameters for PSII RC.

\begin{tabular}{|c|c|c|c|c|c|c|c|}
\hline band & SDF & Oscillator & Excited & $\mathrm{S}_{\mathrm{PSB}}$, & $\omega_{\mathrm{m}} ;$ & $\lambda_{0}$ & $\sigma$ \\
& peak; & strength & state & $\mathrm{S}_{80}$ & $\Gamma_{\text {Gauss }}$ & & \\
& width & (Chl eq. $)$ & lifetime & & $\Gamma_{\text {Lorentz }}$ & & \\
& $\left(\mathrm{cm}^{-1}\right)$ & & $(\mathrm{ns})$ & & $\left(\mathrm{cm}^{-1}\right)$ & & \\
\hline 684 & $14605 ;$ & 2 & 6.0 & $0.80 \pm 0.05$ & $17 ; 11 ; 17$ & $10.4 \pm 0.2$ & $1.1 \pm 0.2$ \\
$\mathrm{~nm}$ & 90 & & & 0.2 & & & \\
\hline $680^{+}$ & $14699 ;$ & $2 *$ & 6.0 & $0.80 \pm 0.05$ & $17 ; 11 ; 17$ & $10.4 \pm 0.2$ & $1.1 \pm 0.2$ \\
$\mathrm{~nm}$ & 110 & & & 0.2 & & & \\
\hline
\end{tabular}

${ }^{+}$the parameters of the $680 \mathrm{~nm}$ band do not affect burning at $686 \mathrm{~nm}$ significantly, and no attempt was made to vary them to achieve a better fit.

*additionally, the amplitude of the $680 \mathrm{~nm}$ band is three times larger than for the $684 \mathrm{~nm}$ band due to sample heterogeneity, not different oscillator strength.

The pure dephasing-limited homogeneous line width was set to $0.033 \mathrm{~cm}^{-1}(1 \mathrm{GHz})^{28}$. 


\section{References:}

1. Personov, R. I., Alshits, E. I., Bykovskaya, L. A., Opt. Commun. 1972, 6, $169-$ 173.

2. Gorokhovskii, A. A., Kaarli, R. K., Rebane, L.A., JETP Lett. 1974, 20, $216-217$.

3. Völker, S. Annu. Rev. Phys. Chem. 1989, 40, 499-530.

4. van der Laan, H., De Caro, C., Schmidt, Th., Visschers, R. W., van Grondelle, R., Fowler, G. J. S., Hunter, C. N., Völker, S., Chem. Phys. Lett. 1993, 212, 569-580.

5. Groot, M. L., Dekker, J. P., van Grondelle, R., den Hartog, F. T. H., Völker, S., J. Phys. Chem. 1996, 100, 11488-11495.

6. Rätsep, M., Blankenship, R. E., Small, G. J., J. Phys. Chem. B 1999, 103, 57365741.

7. Wu, H.-M., Savikhin, S., Reddy, N. R. S., Jankowiak, R., Cogdell, R. J., Struve, W. S., Small, G. J., J. Phys. Chem. 1996, 100 12022-12033.

8. Reinot, T., Zazubovich, V., Hayes, J. M., Small, G. J., J. Phys. Chem. B 2001 105, 5083-5098.

9. Rebane, K., Impurity Centers in Solids, Plenum, NY, 1970

10. Riley, K., Jankowiak, R., Rätsep, M., Small, G. J., Zazubovich, V., J. Phys. Chem. B 2004, 108, 10346-10356.

11. Prokhorenko, V. I.; Holzwarth, A. R., J. Phys. Chem. B 2000, 104, 11563-11578.

12. Romero, E., van Stokkum, I. H. M., Novoderezhkin, V. I., Dekker, J. P., van Grondelle, R., Biochemistry 2010, 49, 4300-4307.

13. Myers, J.A., Lewis, K. L. M., Fuller, F. D., Tekavac, P. F., Yocum, C. F., Ogilvie, J. P., J. Phys. Chem. Lett. 2010, 1, 2774-2780.

14. Reinot, T., Small, G. J., J. Chem. Phys. 2001, 114, 9105-9114.

15. Hayes, J. M., Lyle, P. A.; Small, G. J. J. Phys. Chem. 1994, 98, 7337-7341.

16. Grozdanov, D., Herascu, N., Reinot, T., Jankowiak, R., Zazubovich, V., J. Phys. Chem. B 2010, 114, 3426-3438.

17. Jang, S., Newton, M. D., Silbey, R. J., Phys. Rev. Lett. 2004, 92, 218301. 
18. Jordan, P., Fromme, P., Witt, H. T., Klukas, O., Saenger, W., Krau $\beta$, N., Nature 2001, 411, 909-917.

19. Loll, B., Kern, J., Zouni, A., Saenger, W., Biesiadka, J., Irrgang, K.-D., Photosynth. Res. 2005, 86, 175-184.

20. Ben-Shem, A.; Frolow, F.; Nelson, N. Nature 2003, 426, 630-635.

21. McDermott, G.; Prince, S. M.; Freer, A. A.; Hawthornthwarte-Lawless, A. M.; Papiz, M. Z.; Cogdell, R. J.; Isaacs, N. W. Nature 1995, 374, 517-521.

22. Sener, M. K., Lu, D., Ritz, T., Park, S., Fromme, P., Schulten, K., J. Phys. Chem. B 2002, 106, 7948-7960.

23. Damjanovic, A.; Vaswani, H. M.; Fromme, P.; Fleming, G. R. J. Phys. Chem. B 2002, 106, 10251-10262.

24. Vasil'ev, S.; Orth, P.; Zouni, A.; Owens, T. G.; Bruce, D. Proc. Natl. Acad. Sci. U.S.A. 2001, 98, 8602-8607.

25. Alden, R. G., Johnson, E., Nagarajan, V., Parson, W. W., Law, C. J., Cogdell, R. G., J. Phys. Chem. B 1997, 101, 4667-4680.

26. Sauer, K., Cogdell, R. J., Prince, S. M., Freer, A. A., Isaacs, N. W., Scheer, H.,. Photochem. Photobiol. 1996, 64, 564-576.

27. Pearlstein R. M. Photosynth. Res. 1992, 31, 213-226.

28. Jankowiak, R., Zazubovich, V., Rätsep, M., Matsuzaki, S., Alfonso, M., Picorel, R., Seibert, M., Small, G. J., J. Phys. Chem. B 2000, 104, 11805-11815.

29. Di Donato, M.; van Grondelle, R.; van Stokkum, I. H.; Groot, M. L. J. Phys. Chem. B 2007, 111, 7345-7352.

30. Riley, K. J., Reinot, T., Jankowiak, R., Fromme, P., Zazubovich, V., J. Phys. Chem. B 2007, 111, 286-292.

31. Zazubovich, V.; Matsuzaki, S.; Johnson, T. W.; Hayes, J. M.; Chitnis, P. R.; Small, G. J., Chem. Phys. 2002, 275, 47-59.

32. Byrdin, M., Jordan, P., Krau $\beta$, N. Fromme, P., Stehlik, D., Schlodder, E., Biophys. J. 2002, 83, 433-457.

33. Elli, A. F., Jelezko, F., Tietz, C., Studier, H., Brecht, M., Bittl, R., Wrachtrup, J., Biochemistry 2006, 45, 1454-1458.

34. Scholes, G. D., Fleming, G. R., J. Phys. Chem. B 2000, 104, 1854-1868. 
35. Mukai, K., Abe, S., Sumi, H., J. Phys. Chem. B 1999, 103, 6096-6102.

36. van Oijen, A. M., Ketelaars, M., Köhler, J., Aartsma, T. J., Schmidt, J., Biophys. J. 2000, 78, 1570-1577.

37. Baier, J., Richter, M. F., Cogdell, R. J., Oellerich, S., Köhler, J., J. Phys. Chem. B 2007, 111, 1135-1138.

38. de Weerd, F. L., van Stokkum, I. H. M., van Amerongen, H., Dekker, J. P., van Grondelle, R., Biophys. J. 2002, 82, 1586-1597.

39. Greenfield, S. R., Seibert, M., Wasielewski, M., J. Phys. Chem. B 1999, 103, 8364-8374.

40. Merry, S. A. P., Kumazaki, S., Tachibana, Y., Joseph, D. M., Porter, G., Yoshihara, K., Barber, J., Durrant, J. R., Klug, D. R., J. Phys. Chem. 1996, 100, 10469-10478.

41. Groot, M. L., Pawlowicz, N. P., van Wilderen, L. J. G. W., Breton, J., van Stokkum, I. H. M., van Grondelle, R., Proc. Natl. Acad. Sci. USA 2005, 102, 13087-13092.

42. Salverda, J. M., Vengris, M., Krueger, B. P., Scholes, G. D., Czarnoleski, A. R., Novoderezhkin, V., van Amerongen, H., van Grondelle, R., Biophys. J. 2003, 84, 450-465.

43. Gibasiewicz, K., Croce, R., Morosinotto, T., Ihalainen, J. A., van Stokkum, I. H. M., Dekker, J. P., Bassi, R., van Grondelle, R., Biophys. J. 2005, 88, 1959-1969.

44. Stahl, A. D., Di Donato, M., van Stokkum, I. H. M., van Grondelle, R., Groot, M. L., Biophys. J. 2009, 97, 3215-3223.

45. Holzwarth, A. R., Müller, M. G., Reus, M., Nowaczyk, M., Sander, J., Rögner, M., Proc. Natl. Acad. Sci. USA 2006, 103, 6895-6900.

46. Croce, R., Müller, M. G., Bassi, R., Holzwarth, A. R., Biophys. J. 2003, 84, 25082516.

47. Reinot, T., Small, G. J., J. Chem. Phys. 2000, 113, 10207-10214.

48. Herascu, N., Najafi, M., Amunts, A., Pieper, J., Irrgang, K.-D., Picorel, R., Seibert, M., Zazubovich, V., J. Phys. Chem. B 2011, 115, 2737-2747.

49. Dang, N. C., Reinot, T., Reppert, M., Jankowiak, R., J. Phys. Chem. B, 2007, 111, 1582-1589. 
50. Reinot, T., Hayes, J. M., Small, G. J., J. Chem. Phys.1999, 110, 4820-4827.

51. Reinot, T., Dang, N. C., Small G. J., J. Chem. Phys. 2003, 119, 10404-10414.

52. Rätsep, M.; Johnson, T. W., Chitnis, P. R., Small, G. J., J. Phys. Chem. B 2000, 104, 836-847.

53. Reinot, T., Dang, N. C., Small, G. J., J. Lumin. 2002, 98, 183-188.

54. Dang, N. C., Zazubovich, V., Reppert, M., Neupane, B., Picorel, R., Seibert, M., Jankowiak, R., J. Phys. Chem. B 2008, 112, 9921-9933.

55. Du, H., Fuh, R. A., Li, J., Corkan, A., Lindsey, J. S., Photochem. Photobiol. 1998, 68, 141-142.

56. Sumi, H., J. Phys. Chem. B 1999, 103, 252-260.

57. Sumi, H., Chemical Record 2001, 1, 480-493.

58. Pishchalnikov, R. Y., Müller, M. G., Holzwarth, A. R., in Photosynthesis. Energy from the Sun: 14th International Congress on Photosynthesis, J. F. Allen, E. Gantt, J. H. Golbeck, and B. Osmond (eds.), Springer 2008, pp 163-166.

59. Novoderezhkin, V. I., van Grondelle, R., Phys. Chem. Chem. Phys. 2010, 12, $7352-7365$.

60. Förster, T, Ann. Physik 1948, 437, 55-75.

61. Zazubovich, V., Jankowiak, R., J. Lumin. 2007, 127, 245-250.

62. Riley, K. J., Zazubovich, V., Jankowiak, R., J. Phys. Chem. B 2006, 110, 2243622446.

63. Reppert, M., Zazubovich, V., Dang, N. C., Seibert, M., Jankowiak, R., J. Phys. Chem. B, 2008, 112, 9934-9947.

64. Ishizaki, A., Fleming, G. R., J. Chem. Phys. 2009, 130, 234110.

65. Ishizaki, A., Fleming, G. R., J. Chem. Phys. 2009, 130, 234111.

66. Yang, M., Fleming, G. R., Chem. Phys. 2002, 275, 355-372.

67. Novoderezhkin, V. I., Palacios, M. A., van Amerongen, H., van Grondelle, R., J. Phys. Chem. B 2004, 108, 10363-10375.

68. Kolaczkowski, S. V, Hayes, J. M., Small, G. J., J. Phys. Chem. 1994, 98, 1341813425.

69. Gillie, J. K.; Small, G. J.; Golbeck, J. H., J. Phys. Chem. 1989, 93, 1620-1627.

70. Zazubovich, V., Tibe, I., Small, G. J., J. Phys. Chem. B, 2001, 105, 12410-12417. 
71. Wendling, M.; Pullerits, T.; Przyjalowski, M. A.; Vulto, S. I. E.; Aartsma, T. J.; van Grondelle, R.; van Amerongen, H. J. Phys. Chem. B, 2000, 104, 5825-5831.

72. Peterman, E. J. G.; Pullerits, T.; van Grondelle, R.; van Amerongen, H. J. Phys. Chem. B, 1997, 101, 4448-4457.

73. Rätsep, M, Pieper, J., Irrgang, K.-D., Freiberg, A., J. Phys. Chem. B, 2008, 112, 110-118.

74. Hughes, J. L., Picorel, R., Seibert, M., Krausz, E., Biochemistry 2006, 45, $12345-$ 12357.

75. Groot, M.-L., Frese, R., N., de Weerd, F. L., Bromek, K., Petersson, Á., Peterman, E. J. G., van Stokkum, I. H. M., van Grondelle, R., Dekker, J. P., Biophys. J. 1999, 77, 3328-3340.

76. Krausz, E., Hughes J. L., Smith, P., Pace, R., Peterson Ársköld, S., Photochem. Photobiol. Sci. 2005, 4, 744-753.

77. Takahashi, Y., Hansson, O., Mathis, P., Satoh, K. Biochim. Biophys. Acta 1987, 893, 49-59.

78. Groot, M.-L., Peterman, E. J. G., van Kan, P. J. M., van Stokkum, I. H. M., Dekker, J. P., van Grondelle, R., Biophys. J. 1994, 67, 318-330. 


\section{Figure Captions}

Figure 1: Frame A: dispersive HGK, calculated using Eqs. 1-3 with $\Omega=\omega_{B}$, for $\lambda_{B}$ at the peak/center of the SDF. $\mathrm{S}=0.6$; the homogeneous $Z \mathrm{PL}$ width was set to $3 \mathrm{~cm}^{-1}$. The distribution of tunneling parameter $\lambda$ peaking at $\lambda_{0}=10$ and had a $\sigma_{\lambda}=0.0$ (no dispersion), $0.3,0.6,1.0$ and 1.5 , corresponding to the black, blue, red, green and purple curves, respectively. Frame B: hole FWHM as a function of the fractional hole depth, color coded the same as in frame A. The insert depicts an example of a theoretical hole spectrum, with arrows indicating that the width was determined at half the maximal depth.

Figure 2: Frame A: HGK curves obtained with fixed $\lambda_{0}=10.0$ and $\sigma_{\lambda}=1.0$ with the Gaussian line width distributions peaking at $3 \mathrm{~cm}^{-1}$ and with widths (FWHM) of 0.0 (no distribution) $0.5,1.0,2.0$, and $3.0 \mathrm{~cm}^{-1}$ corresponding to black, blue, red, green and purple curves, respectively. The insert: beginning of the curves on a nonlogarithmic scale. Frame B: Dependences of the hole FWHM on fractional hole depth for line width distribution parameters as in frame A. Frame C: HGK curves obtained with fixed $\lambda_{0}=10.0$ and $\sigma_{\lambda}=1.0$ and with a Gaussian line width distributions peaking at $0.3 \mathrm{~cm}^{-1}$ and with widths (FWHM) of $0.0,0.05,0.1,0.2$, and $0.3 \mathrm{~cm}^{-1}$ corresponding to black, blue, red, green and purple curves, respectively. Frame D: Dependences of the hole FWHM on fractional hole depth for line width distribution parameters as in frame C. Horizontal dashed lines in frames B and D denote the hole width expected in case of no line width dispersion. 
Figure 3. Frame A: Indistinguishable HGK curves for different combinations of $\lambda$ distribution and line width distribution parameters (see below). Frame B: Easily distinguishable hole width dependences on the hole depth for the same parameters as in frame A. Red curve: line width distribution peaking at $3.0 \mathrm{~cm}^{-1}$ with $\mathrm{FWHM}=3.0$ $\mathrm{cm}^{-1}, \lambda_{\mathrm{o}}=10.0$, and $\sigma_{\lambda}=1.0$. Green: line width distribution peaking at $2.5 \mathrm{~cm}^{-1}$ with $\mathrm{FWHM}=2.1 \mathrm{~cm}^{-1}, \lambda_{\mathrm{o}}=10.15$, and $\sigma_{\lambda}=1.0$. Black: line width distribution peaking at 2.0 $\mathrm{cm}^{-1}$ with FWHM=1.3 $\mathrm{cm}^{-1}, \lambda_{\mathrm{o}}=10.2$, and $\sigma_{\lambda}=1.0$. Blue: single line width $1.5 \mathrm{~cm}^{-1}$, $\lambda_{\mathrm{o}}=10.5$, and $\sigma_{\lambda}=1.0$.

Figure 4: The spectral overlap dependence on ZPL-ZPL energy gap (black curve) for the single site spectra parameters in Table 1, two SDF (solid red and blue curves), and deconvolution of those SDFs into sub-SDFs of fractions capable (dashed line) or incapable (dotted line) of EET. The dash-dotted green curve is the overlap integral dependence on energy gap according to Kolaczkowski et al. ${ }^{68 .}$ Vertical solid arrows indicate contributions to the overlap from localized vibrations; the dashed arrow indicates minimal overlap. The dotted arrow indicates maximal overlap within the range of Figure 5.

Figure 5: Overlap distributions (lower scale) and homogeneous line width distributions with $V_{A B}=5 \mathrm{~cm}^{-1}$ for EET between two bands with the SDFs resembling those of the two lowest-energy states of the CP43 complex (the insert contains its absorption spectrum and two SDFs). The blue curve is the distribution for $\mathrm{A} \rightarrow \mathrm{B}$ EET for $\lambda_{\mathrm{B}}=680 \mathrm{~nm}$, while the red curve is the distribution for $\mathrm{B} \rightarrow \mathrm{A}$ EET. The horizontal 


\section{Page 41 of 51}

dashed arrow indicates the $\sim 20 \%$ contribution of B-type pigments incapable of EET at $\lambda_{\mathrm{B}}$. NB: Homogeneous line width reported in the figure does not include pure dephasing contribution (e.g. $0.033 \mathrm{~cm}^{-1}$ at $5 \mathrm{~K}^{28}$ ).

Figure 6: Frame A: The absorption spectrum of CP43 as well as the SDFs of the lowest-energy states according to various models discussed in the text. Frame B: Experimental dependence of the hole width on hole depth for $\lambda_{\mathrm{B}}=680.15 \mathrm{~nm}$. Black diamonds: high-resolution data. Blue diamonds: low resolution data (see frame D), corrected for the spectrometer resolution. The solid curve is the theoretical dependence for $V_{A \mathrm{~B}}=5 \mathrm{~cm}^{-1}$, and the dashed curve is the theoretical dependence for $V_{A B}=10 \mathrm{~cm}^{-1}$. The dotted curve was obtained in the absence of the line width distribution.

Figure 7: Frame A: the absorption spectrum of isolated PS II RC complex. The arrows indicates the burn wavelengths of 680 and $686 \mathrm{~nm}$, the latter within a weak shoulder at $\sim 684 \mathrm{~nm}$. Frame B: persistent hole depth versus irradiation dose data for $\lambda_{B}=686 \mathrm{~nm}$. The noisy curves are HGK curves; symbols refer to data extracted from the hole spectra. The numbers refer to the intensity $\left(\right.$ in $\mathrm{mW} / \mathrm{cm}^{2}$ ) used to obtain the data. The fastest two HGK (red and magenta) were obtained in fluorescence excitation mode. Also presented is the best fit (solid black line) to the lowest-intensity transmission mode data (noisy green curve + open circles). See the text for line width distribution details. Frame C: experimental persistent hole width vs depth data (open and solid circles represent two complimentary data sets) for $\lambda_{B}=686 \mathrm{~nm}$, and 
theoretical fits with the same distribution as in Frame B (solid curve), with the distribution from ${ }^{11}$ (dashed curve) and without any line width distribution (dotted curve). The solid triangles represent the experimental data obtained at $680 \mathrm{~nm}$. 



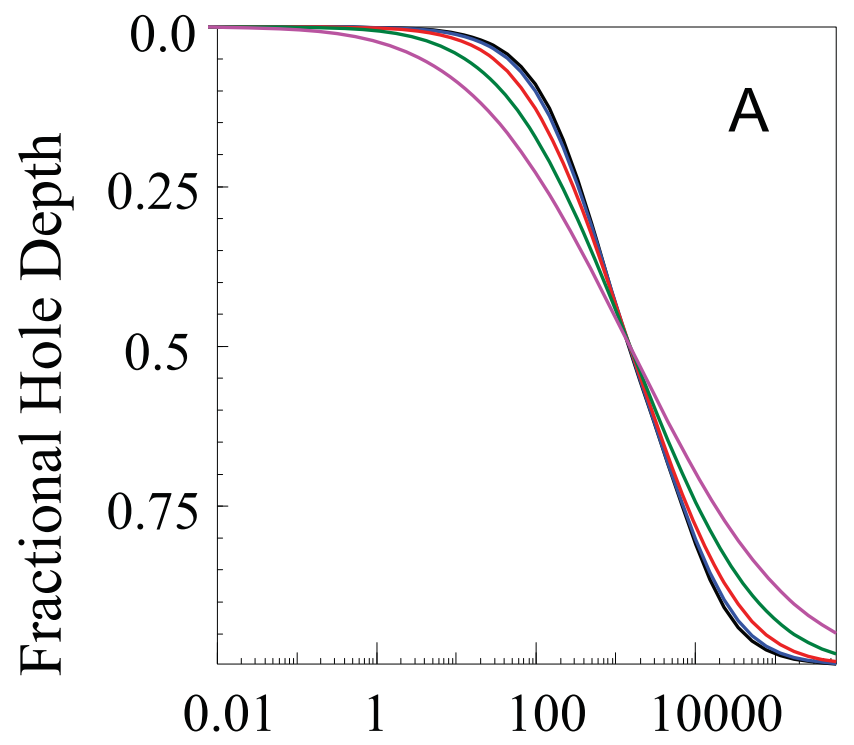

Irradiation Dose $\left(\mathrm{J} / \mathrm{cm}^{2}\right)$

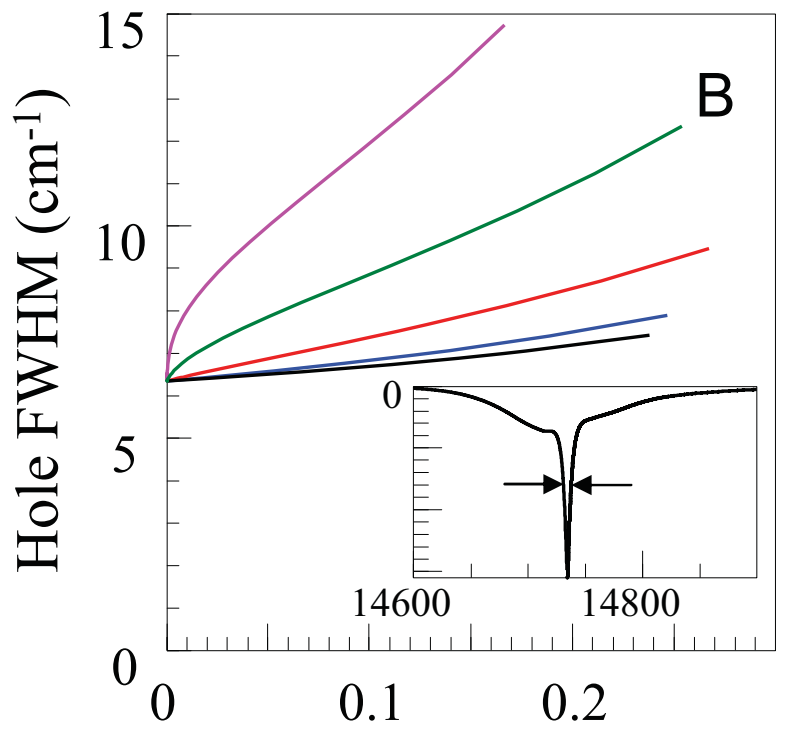

Fractional Hole Depth

Figure 1. 


\section{Page 45 of 51}
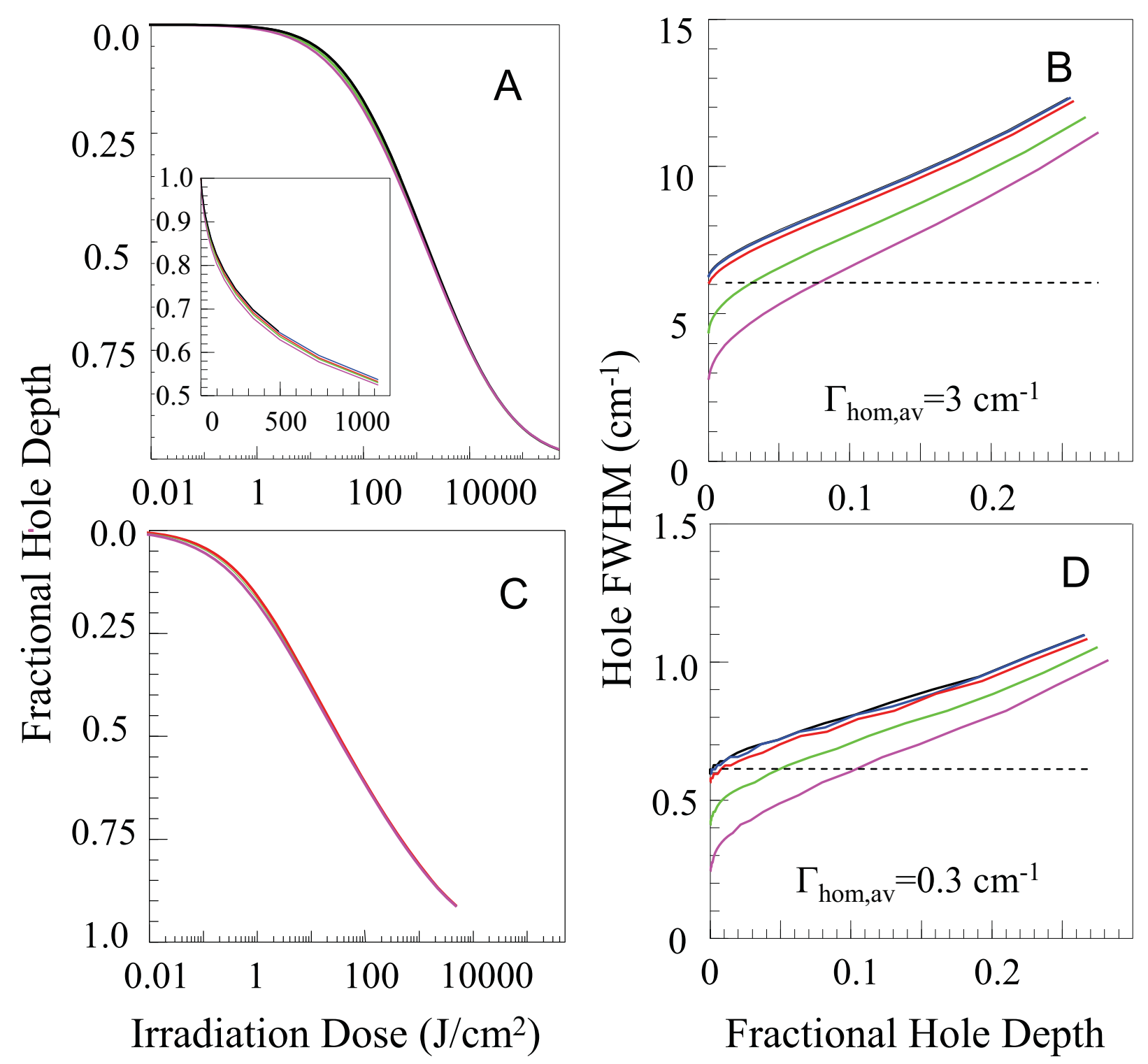

Figure 2 

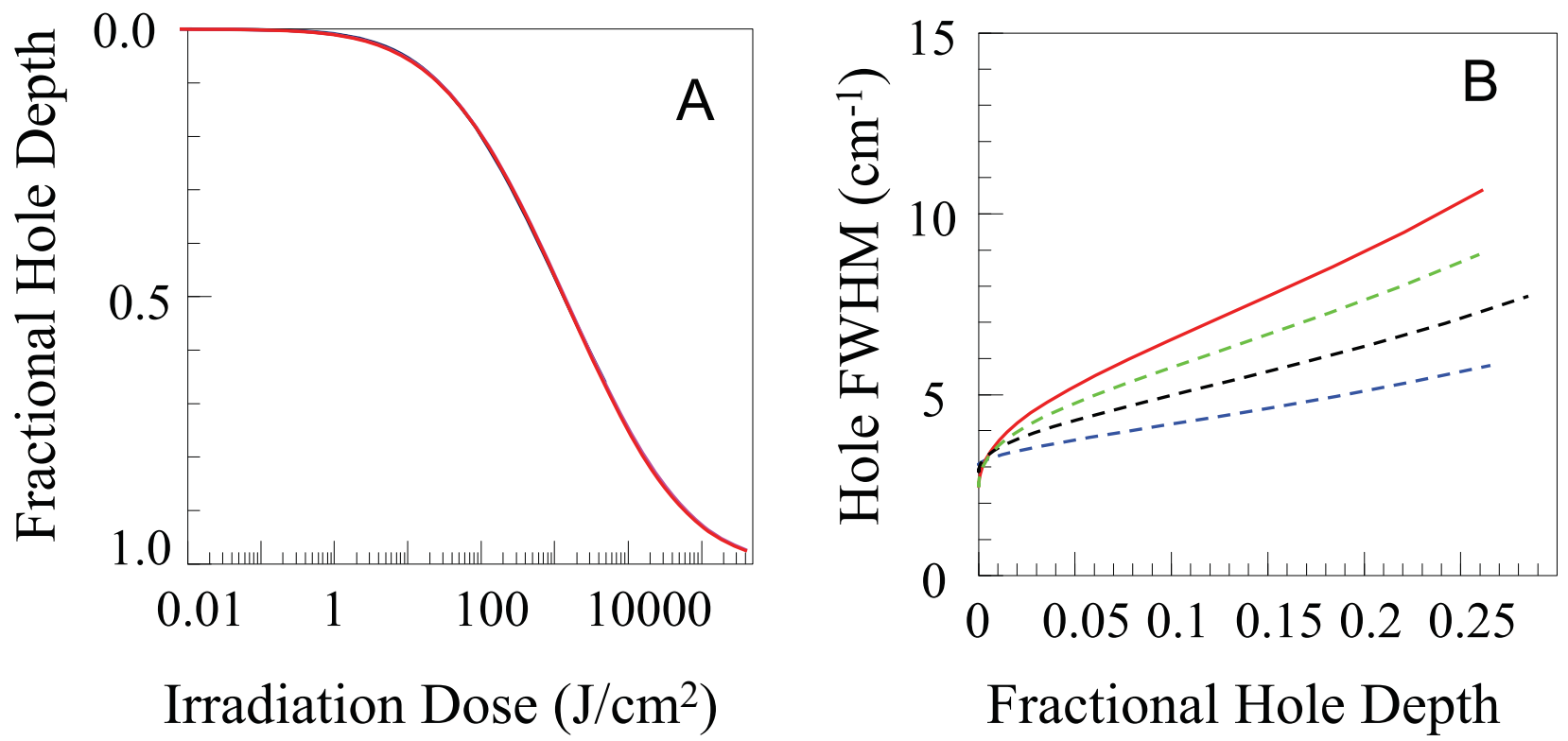

Fractional Hole Depth

Figure 3 


\section{Page 47 of 51}

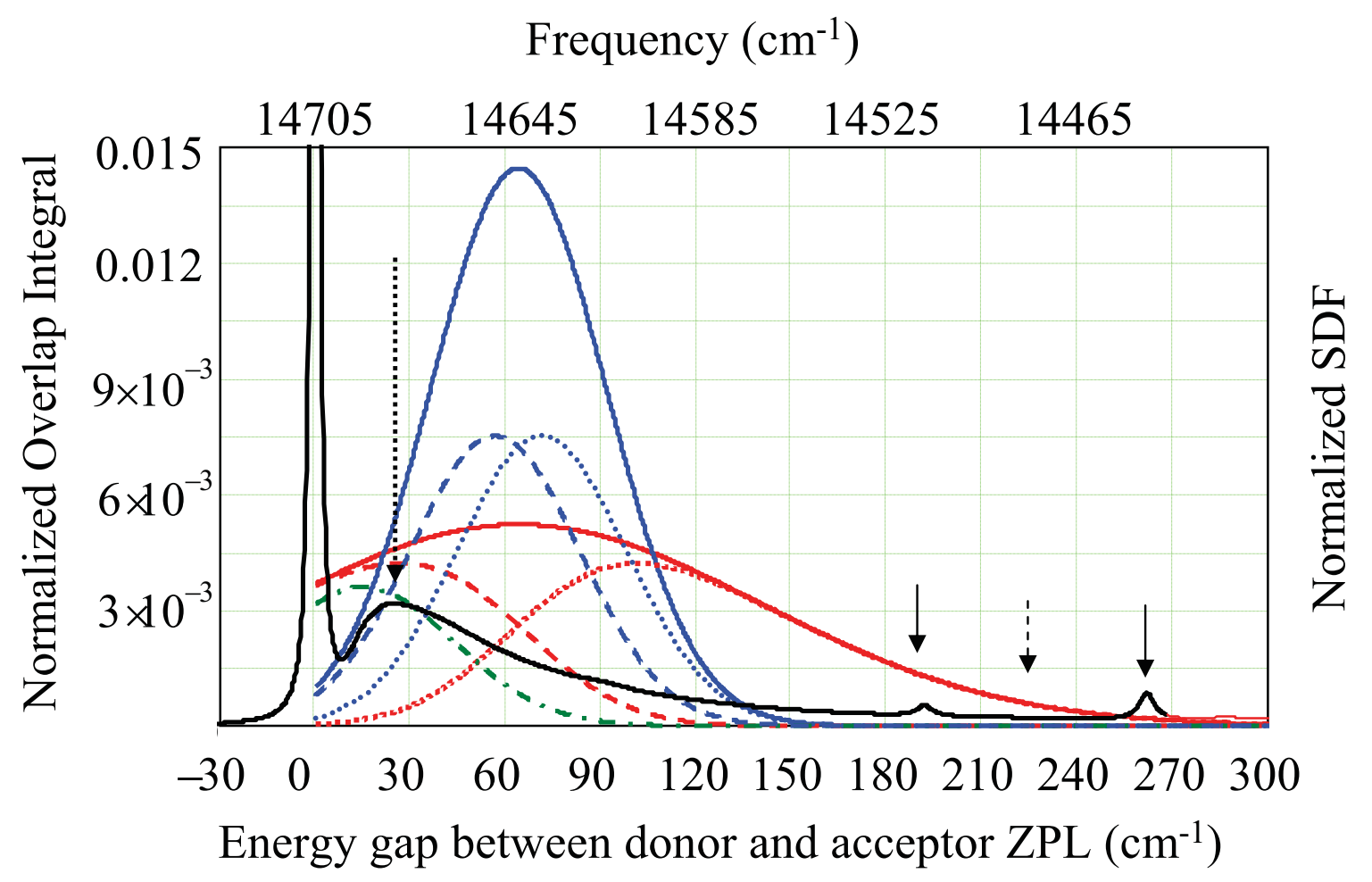

Figure 4 


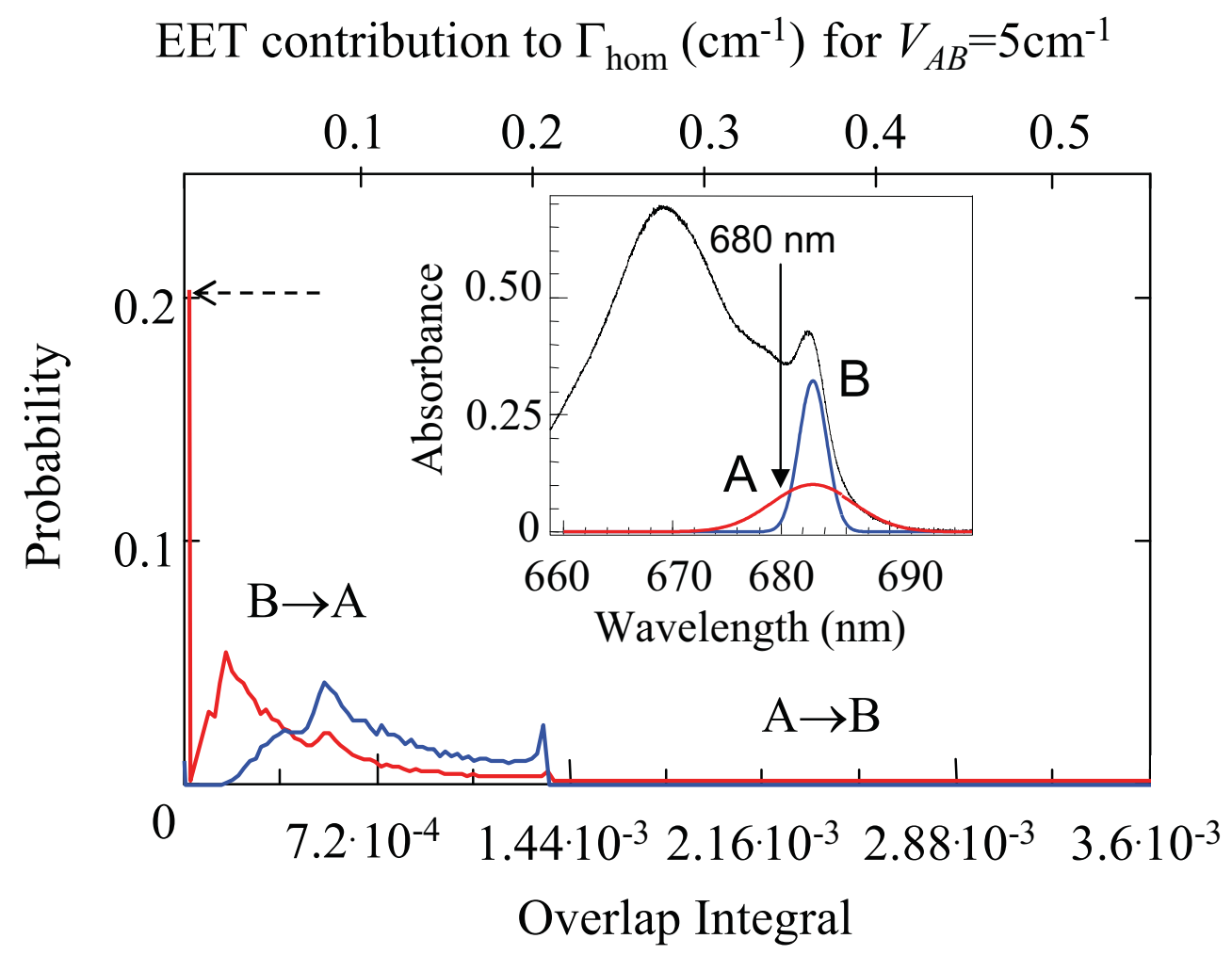

Figure 5 


\section{Page 49 of 51}
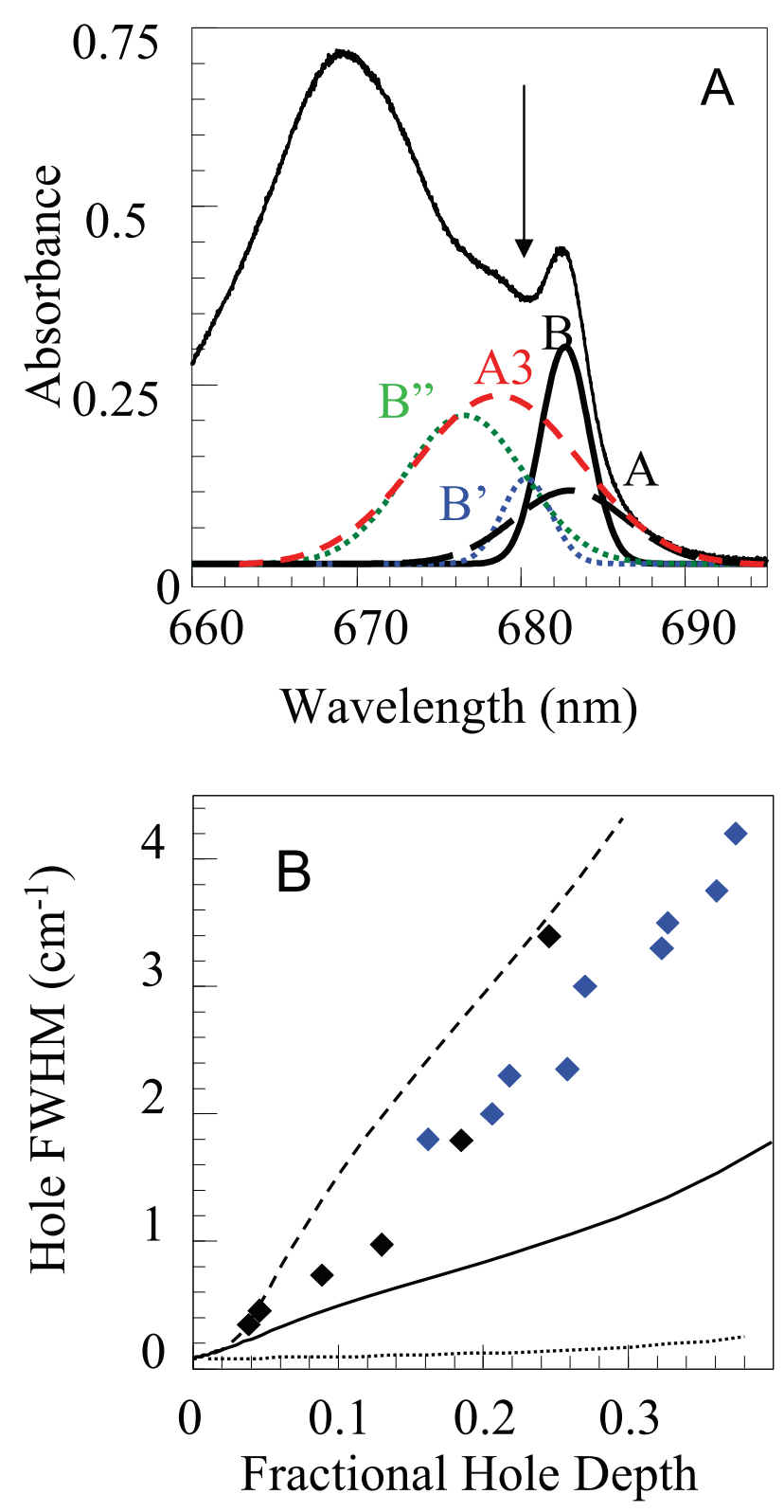

Figure 6 

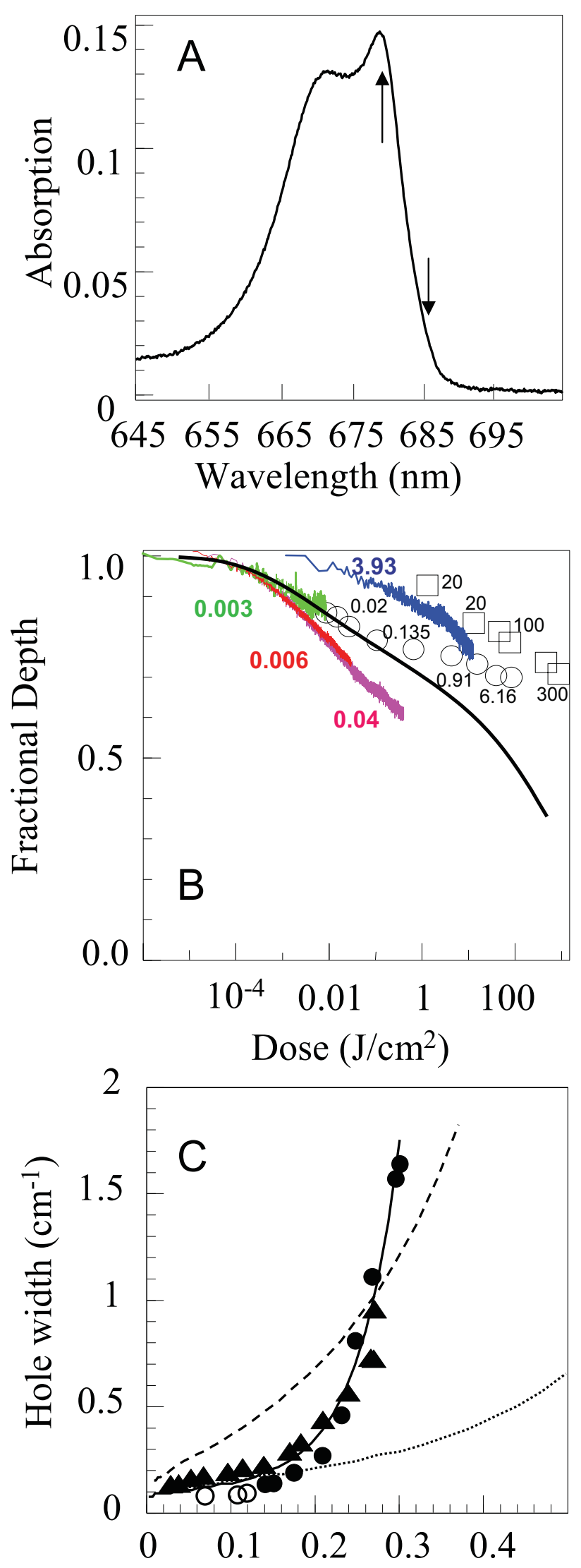

Fractional depth

Figure 7 


\section{Page 51 of 51}

\section{Graphical Abstract}

